\title{
The Piecewise Cubic Method (PCM) for Computational Fluid Dynamics
}

\author{
Dongwook Lee ${ }^{\mathrm{a}, *}$, Hugues Faller ${ }^{\mathrm{a}, \mathrm{c}}$, Adam Reyes $^{\mathrm{b}}$ \\ ${ }^{a}$ Applied Mathematics and Statistics, University of California, Santa Cruz, CA, U.S.A \\ ${ }^{b}$ Department of Physics, University of California, Santa Cruz, CA, U.S.A \\ ${ }^{c}$ Département de Physique, École Normale Supérieure, Paris, France
}

\begin{abstract}
We present a new high-order finite volume reconstruction method for hyperbolic conservation laws. The method is based on a piecewise cubic polynomial which provides its solutions a fifth-order accuracy in space. The spatially reconstructed solutions are evolved in time with a fourth-order accuracy by tracing the characteristics of the cubic polynomials. As a result, our temporal update scheme provides a significantly simpler and computationally more efficient approach in achieving fourth order accuracy in time, relative to the comparable fourth-order Runge-Kutta method. We demonstrate that the solutions of PCM converges at fifth-order in solving 1D smooth flows described by hyperbolic conservation laws. We test the new scheme on a range of numerical experiments, including both gas dynamics and magnetohydrodynamics applications in multiple spatial dimensions.
\end{abstract}

Keywords: High-order methods; piecewise cubic method; finite volume method; gas dynamics; magnetohydrodynamics; Godunov's method.

\footnotetext{
* Corresponding author

Email address: dlee79@ucsc.edu (Dongwook Lee)
} 


\section{Introduction}

In this paper we are interested in solving multidimensional conservation laws of the Euler equations and the ideal MHD equations, written as

$$
\frac{\partial \mathbf{U}}{\partial t}+\nabla \cdot \mathcal{F}(\mathbf{U})=0
$$

where $\mathbf{U}$ is the vector of the conservative variables, and

$$
\mathcal{F}(\mathbf{U})=[F(\mathbf{U}), G(\mathbf{U}), H(\mathbf{U})]^{T}=[\mathbf{F}, \mathbf{G}, \mathbf{H}]^{T}
$$

5 is the flux vector.

We present a new high-order piecewise cubic method (PCM) algorithm that is extracted from the classical PPM [1] and WENO schemes [2, 3]. These two algorithms, by far, have been extremely successful in various scientific fields where there are challenging computational needs for both high-order accuracy in smooth flows and well-resolved solutions in shock/discontinuous flows. With the advent of high-performance computing (HPC) in recent years, such needs have been more and more desired, and have become a necessary requirement in conducting large scale, cutting edge simulations of gas dynamics and magnetohydrodynamics (MHD) [4, 5, 6, 7.

As observed in the success stories of the PPM and WENO methods, discrete algorithms of data interpolation and reconstruction play a key role in numerical methods for PDE approximations [8, 9, 10] within the broad framework of finite difference and finite volume discretization methods. In view of this, computational improvements of such interpolation and reconstruction schemes, particularly focused on the high-order property with great shock-capturing capability, take their positions at the center of HPC in modern computational fluid dynamics.

The properties of enhanced solution accuracy with lower numerical errors on a given grid resolution and faster convergence-to-solution rates are the key 25 advantages in high-order schemes. The advantage of using high-order methods in HPC is therefore clear: one can obtain reproducible, admissible, and highly 
accurate numerical solutions in a faster computational time at the expense of increased rate of floating point operations, while at the same time, with the use of smaller size of grid resolutions. This is by no means exceedingly efficient in high-performance computing (HPC), in view of the fact that the increase of grid resolutions has a direct impact to an increase of memory footprints which are bounded in all modern computing architecture.

In this regards, our goal in this paper is to lay down a mathematical foundation in designing a new high-order method using piecewise cubic polynomials.

35 For the scope of the current paper we focus on describing the detailed PCM algorithm in 1D finite volume framework in which a fourth order PCM reconstruction is formulated in each normal dimension without cross derivative terms. For multidimensional problems we adopt the classical $\ddagger$ dimension-bydimension 12 approach for simplicity, where a fourth order PCM reconstruc-

40 tion in each normal direction is used to compute second order time averaged normal fluxes. The multidimensional conservation laws are updated using a directionally unsplit fashion. Although this approach has an advantage in its simplicity, it unfortunately fails to retain the high-order accurate property of the $1 \mathrm{D}$ baseline algorithm in multidimensional problems. Instead, it provides only a second-order accuracy in multidimensional nonlinear advection in finite volume method due to the lack of accuracy in approximating a face-averaged flux function as a result of mis-using an averaged quantity in place of a pointwise quantity, or vice versa [11, 12, 13, 14]. We understand that the baseline 1D PCM scheme can be extended to multiple spatial dimensions preserving its high-order accuracy by following more sophisticated treatments in two different ways. A first approach can be a traditional approach of combining high-order spatial reconstructions and high-order Runge-Kutta (RK) temporal updates [11, 12, 13, 14]. Alternatively, a second approach can be available with more

\footnotetext{
$\ddagger$ This approach is the same as the Class A approach in [13], and should not be confused with the so-called dimensionally split approach. Our spatial integration scheme in this paper is directionally unsplit.
} 
computationally efficient formulations based on one-step ADER formulations 15, 16, 17, 18, 19, 20, 21, 22, to extend the baseline 1D PCM scheme to multiple spatial dimensions with high-order accuracy. The ADER methods have shown great promises in achieving high-order accurate solutions in multiple spatial dimensions using efficient temporal updating strategies for structured and unstructured grid geometries [19, 23, as well as for adaptive mesh refinement geometry 20]. As for the computational performance, the ADER schemes can yield about an order of magnitude improved time-to-solution performance relative to the RK integration methods of the same solution accuracy [21]. Such a work will be considered in our future research to deliver high-oder accuracy in designing the PCM scheme multidimensionally.

For a finite volume scheme in $1 \mathrm{D}$ we take the spatial average of Eq. (1) over the cell $I_{i}=\left[x_{i-1 / 2}, x_{i+1 / 2}\right]$, yielding a semi-discrete form,

$$
\frac{\partial \overline{\mathbf{U}}_{i}}{\partial t}=-\frac{1}{\Delta x}\left(\mathbf{F}_{i+1 / 2}-\mathbf{F}_{i-1 / 2}\right)
$$

to get an equation for the evolution of the volume averaged variables, $\overline{\mathbf{U}}_{i}=$ $\frac{1}{\Delta x} \int_{I_{i}} \mathbf{U}(x, t) d x$. Typically to achieve high-order accuracy in time the temporal update is done using a TVD Runge-Kutta scheme in method-of-lines form [24, 14. In this approach the high-order accuracy comes from taking the multiple Euler stages of the RK time discretizations, which require repeated reconstructions in a single time step, increasing the computational cost.

Instead, as will be fully described in Section 2, one of the novel ideas in PCM is to employ the simple single stage predictor-corrector type temporal update formulation in which we take the time-average of Eq. (3)

$$
\overline{\mathbf{U}}_{i}^{n+1}=\overline{\mathbf{U}}_{i}^{n}-\frac{\Delta t}{\Delta x}\left(\mathbf{F}_{i+1 / 2}^{n+1 / 2}-\mathbf{F}_{i-1 / 2}^{n+1 / 2}\right)
$$

Here $\overline{\mathbf{U}}_{i}^{n}=\overline{\mathbf{U}}_{i}\left(t^{n}\right)$ is the volume averaged quantity at $t^{n}$, and $\mathbf{F}_{i \pm 1 / 2}^{n+1 / 2}=$ $\frac{1}{\Delta t} \int_{t^{n}}^{t^{n+1}} \mathbf{F}_{i \pm 1 / 2}(t) d t$ is the time average of the interface flux from $t^{n}$ to $t^{n+1}$. In this way high-order in space and time is accomplished with a single reconstruction in contrast to the multiple Euler stages of the RK time discretizations, 80 providing significant benefits in computational efficiency per solution accuracy. 
The organization of the paper is as follows: Section 2 describes the fifth-order accurate spatial reconstruction algorithm of PCM in 1D. We highlight several desirable properties of the PCM scheme in terms of computational efficiency and solution accuracy. Section 3 introduces the fourth-order accurate temporal updating scheme of PCM using a predictor-corrector type characteristic tracing, which is much simpler than the typical high-order Runge-Kutta ODE updates. In Section 5 we discuss how we extend the 1D scheme in Section 2 to multiple spatial dimensions following the dimension-by-dimension [12, 13] approach to reconstruction, meaning that the PCM reconstruction uses one-dimensional reconstructions in each normal direction, followed by evaluating 1D-based fluxes using a single-point quadrature rule at each cell face, followed by updating the system of multidimensional equations in Eq. (1) in a directionally unsplit fashion.

In Section 6 we test the PCM scheme on a wide spectrum of benchmark problems in 1D, 2D and 3D, both for hydrodynamics and magnetohydrodynamics (MHD) applications. We also compare the PCM solutions with PPM and WENO solutions in order to examine numerical accuracy, capability and efficiency in both smooth and shock flow regimes. We conclude our paper in Section 7 with a brief summary.

\section{The One-Dimensional Piecewise Cubic Method (PCM) Spatial Re- construction}

In this section we describe a new PCM scheme in a one-dimensional finite volume formulation for solving hyperbolic conservation laws of hydrodynamics and magnetohydrodynamics. The new PCM scheme is a higher-order extension of Godunov's method [25], bearing its key components in the reconstruction algorithm on the relevant ideas of its high-order predecessors, the PPM scheme [1, the WENO schemes [2, 26, 27, 28, and Hermite-WENO schemes 29, 30, 31, 32.

For the purpose of this section, we take the $3 \times 3$ hyperbolic system of 
conservation laws of the 1D Euler equations

$$
\frac{\partial \mathbf{U}}{\partial t}+\frac{\partial F(\mathbf{U})}{\partial x}=0
$$

The notations used are the vector of the conservative variables $\mathbf{U}$ and fluxes $F(\mathbf{U})$, respectively, defined as

$$
\mathbf{U}=\left[\begin{array}{c}
\rho \\
\rho u \\
E
\end{array}\right], \quad F(\mathbf{U})=\left[\begin{array}{c}
\rho u \\
\rho u^{2}+p \\
u(E+p)
\end{array}\right] .
$$

Here $\rho$ is the fluid density, $u$ is the fluid velocity in $x$-direction, and $E$ is the total energy as the sum of the internal energy $\epsilon=p /(\gamma-1)$ and the kinetic energy obeying the ideal gas law,

$$
E=\frac{p}{\gamma-1}+\frac{\rho u^{2}}{2}
$$

where $p$ is the gas pressure, with the ratio of specific heats denoted as $\gamma$. We denote the cells in $x$-direction by $I_{i}=\left[x_{i-1 / 2}, x_{i+1 / 2}\right]$. We assume our grid is configured on an equidistant uniform grid for simplicity.

In addition to the system of the Euler equations in the conserved variables $\mathbf{U}$ as given in Eq. (5), we often use the two other equivalent system of equations each of which can be written either in the primitive variables $\mathbf{V}=[\rho, u, p]^{T}$ or in the characteristic variables $\mathbf{W}$. The characteristic variable $\mathbf{W}$ is readily obtained from $\mathbf{U}$ or $\mathbf{V}$ by multiplying the left eigenvectors corresponding to either $\mathbf{U}$ or $\mathbf{V}$, for instance, $\mathbf{W}=\mathbf{L} \mathbf{U}$. In the latter $\mathbf{L}\left(\equiv \mathbf{R}^{-1}\right)$ represents the $3 \times 3$ matrix obtained from diagonalizing the coefficient matrix $\mathbf{A}=\partial \mathcal{F} / \partial \mathbf{U}=\mathbf{R} \mathbf{\Lambda} \mathbf{R}^{-1}$, whose rows are the $k$-th left eigenvectors $\ell^{(k)}, k=1,2,3$. The representation of the system in $\mathbf{W}$ furnishes a completely linearly decoupled 1D system of equations,

$$
\frac{\partial \mathbf{W}}{\partial t}+\boldsymbol{\Lambda} \frac{\partial \mathbf{W}}{\partial x}=0
$$

The above system in the characteristic variables $\mathbf{W}$ is therefore very handy for 130 analyses, and also is a preferred choice of variable in order to furnish numerical solutions more accurate than third-order especially with better non-oscillatory 
controls, in particular when considering wave-by-wave propagations in a system of equations [11. For this reason the characteristic variable $\mathbf{W}$ is taken as our default variable choice in the 1D PCM reconstruction steps via characteristic decompositions, albeit with an increased computational cost, among the other two choices of the primitive $\mathbf{V}$ or the conservative variables $\mathbf{U}$.

The methodology presented below can be similarly applied to the 1D ideal MHD equations (see for instance, [33]).

\subsection{Piecewise Cubic Profile}

To begin with we first define a cubic polynomial $p_{i}(x)$ to approximate a $k$-th characteristic variable $q \in \mathbf{W}$ on each interval $I_{i}$ by

$$
p_{i}(x)=c_{0}+c_{1}\left(x-x_{i}\right)+c_{2}\left(x-x_{i}\right)^{2}+c_{3}\left(x-x_{i}\right)^{3} .
$$

The goal is now to determine the four coefficients $c_{i}, i \in \mathbb{Z}, 0 \leq i \leq 3$, which can be achieved by imposing the following four conditions:

$$
\begin{aligned}
\frac{1}{\Delta x} \int_{I_{i}} p_{i}(x) d x & =\bar{q}_{i}, \\
p_{i}\left(x_{i-1 / 2}\right) & =q_{L, i}, \\
p_{i}\left(x_{i+1 / 2}\right) & =q_{R, i}, \\
p_{i}^{\prime}\left(x_{i}\right) & =q_{C, i}^{\prime},
\end{aligned}
$$

where

$$
\bar{q}_{i}=\frac{1}{\Delta x} \int_{I_{i}} q\left(x, t^{n}\right) d x
$$

is the cell-averaged quantity at $t^{n}$ on $I_{i}$ which is given as an initial condition;

$$
q_{L, i}=q\left(x_{i-1 / 2}, t^{n}\right)+\mathcal{O}\left(\Delta x^{p}\right), \quad q_{R, i}=q\left(x_{i+1 / 2}, t^{n}\right)+\mathcal{O}\left(\Delta x^{p}\right)
$$

are respectively the $p$-th order accurate pointwise left and the right Riemann states at $t^{n}$ on the cell $I_{i}$ that are unknown yet but are to be determined as described below; and lastly

$$
q_{C, i}^{\prime}=q^{\prime}\left(x_{i}, t^{n}\right)+\mathcal{O}\left(\Delta x^{r}\right)
$$


is the $r$-th order accurate approximation to the slope of $q$ at $t^{n}$ evaluated at $x_{i}$, which is again unknown at this point but is to be determined as below.

For the moment let us assume that all four quantities $\bar{q}_{i}, q_{L, i}, q_{R, i}$ and $q_{C, i}^{\prime}$ are known. It can be shown that the system of relations in Eqs. $100 \sim 13$ is equivalent to a system given as:

$$
\begin{aligned}
c_{0}+c_{2} \frac{\Delta x^{2}}{12} & =\bar{q}_{i}, \\
c_{0}-c_{1} \frac{\Delta x}{2}+c_{2} \frac{\Delta x^{2}}{4}-c_{3} \frac{\Delta x^{3}}{8} & =q_{L, i}, \\
c_{0}+c_{1} \frac{\Delta x}{2}+c_{2} \frac{\Delta x^{2}}{4}+c_{3} \frac{\Delta x^{3}}{8} & =q_{R, i}, \\
c_{1} & =q_{C, i}^{\prime},
\end{aligned}
$$

which, in turn, can be solved for all four $c_{i}, i=1, \ldots, 4$. The final expressions of the coefficients in terms of $\bar{q}_{i}, q_{L, i}, q_{R, i}$, and $q_{C, i}^{\prime}$ are given as:

$$
\begin{aligned}
c_{0} & =\frac{1}{4}\left(-q_{R, i}-q_{L, i}+6 \bar{q}_{i}\right), \\
c_{1} & =q_{C, i}^{\prime}, \\
c_{2} & =\frac{3}{\Delta x^{2}}\left(q_{R, i}+q_{L, i}-2 \bar{q}_{i}\right), \\
c_{3} & =\frac{4}{\Delta x^{3}}\left(q_{R, i}-q_{L, i}-\Delta x q_{C, i}^{\prime}\right) .
\end{aligned}
$$

Therefore once we figure out the three unknowns, $q_{L, i}, q_{R, i}$, and $q_{C, i}^{\prime}$, the cubic profile $p_{i}(x)$ in Eq. (9) can be completely determined and is ready to approximate $q$ on each $I_{i}$.

We now devote the following sections to describe how to determine $q_{L, i}, q_{R, i}$, and $q_{C, i}^{\prime}$ so that the resulting PCM approximation to the variable $q$ lend its accuracy a fifth-order in space (Sections 2.2 and 2.3) and a fourth-order in time (Section 3).

\subsection{Reconstruction of the Riemann States $q_{L, i}$ and $q_{R, i}$}

We follow the fifth-order finite volume WENO approach, either of the classical WENO-JS [2], WENO-M [34, or WENO-Z [27, 28], in order to reconstruct 
the left and right Riemann states, $q_{L, i}$ and $q_{R, i}$, on each cell $I_{i}$,

$$
q_{L ; R, i}=\sum_{\ell=1}^{3} \omega_{\ell}^{ \pm} p_{\ell}\left(x_{i \pm 1 / 2}\right) .
$$

where the explicit forms of $p_{\ell}$ are given in Appendix A. The nonlinear weights $\omega_{\ell}^{ \pm}$are determined by using one of the following schemes:

- For WENO-JS:

$$
\omega_{\ell}^{ \pm, J S}=\frac{\tilde{\omega}_{\ell}^{ \pm}}{\sum_{s} \tilde{\omega}_{s}^{ \pm}}, \text {where } \tilde{\omega}_{\ell}^{ \pm}=\frac{\gamma_{\ell}^{ \pm}}{\left(\epsilon+\beta_{\ell}\right)^{m}}
$$

170

- For WENO-M:

$$
\omega_{\ell}^{ \pm, M}=\frac{\tilde{\omega}_{\ell}^{ \pm}}{\sum_{s} \tilde{\omega}_{s}^{ \pm}}
$$

where

$$
\tilde{\omega}_{\ell}^{ \pm}=g_{\ell}\left(\omega_{\ell}^{ \pm, J S}\right)=\frac{\omega_{\ell}^{ \pm, J S}\left(\gamma_{\ell}^{ \pm}+\left(\gamma_{\ell}^{ \pm}\right)^{2}-3\left(\gamma_{\ell}^{ \pm}\right) \omega_{\ell}^{ \pm, J S}+\left(\omega_{\ell}^{ \pm, J S}\right)^{2}\right)}{\left(\gamma_{\ell}^{ \pm}\right)^{2}+\omega_{\ell}^{ \pm, J S}\left(1-2 \gamma_{\ell}^{ \pm}\right)}
$$

- For WENO-Z:

$$
\omega_{\ell}^{ \pm, Z}=\frac{\tilde{\omega}_{\ell}^{ \pm}}{\sum_{s} \tilde{\omega}_{s}^{ \pm}}, \text {where } \tilde{\omega}_{\ell}^{ \pm}=\gamma_{\ell}^{ \pm}\left(1+\left(\frac{\left|\beta_{0}-\beta_{2}\right|}{\epsilon+\beta_{\ell}}\right)^{m}\right)
$$

In the above, the smoothness indicators $\beta_{\ell}$ are given by following the standard fifth order WENO reconstruction approach [11. We also display their explicit forms in Appendix A.

In Eqs. 26), 27), and (29), $\epsilon$ is any arbitrarily small positive number that prevents division by zero, for which we choose $\epsilon=10^{-36}$. One of the classical choice of $\epsilon$ in many WENO literatures is found to be $\epsilon=10^{-6}$ [2, 26]; however, it was suggested in [34, 27] that $\epsilon$ should be chosen to be much smaller in order to force this parameter to play only its original role of avoiding division by zero in the definitions of the weights, Eqs. (26) and 29. 
Another closely related point of discussion is with the value of $m$, the power in the denominators in Eqs. 26) and (29). The parameter $m$ determines the rate of changes in $\beta_{\ell}$, and most of the WENO literatures use $m=2$. However, we observe that using $m=1$ resolves discontinuities sharper in most of our numerical simulations without exhibiting any numerical instability, so became the default value in our implementation. For more detailed discussions on the choices of $\epsilon$ and $m$, see [34, 27, 28.

Using these nonlinear weights, we complete the WENO reconstruction procedure of producing the fifth-order spatially accurate, non-oscillatorily reconstructed values at each cell interface at each time step $t^{n}$ as defined in Eq. (25).

2.3. Reconstruction of the Derivative $q_{C, i}^{\prime}$

The spatial reconstruction part of PCM proceeds to the next final step to obtain the derivative $q_{C, i}^{\prime}$ in Eq. $13 p$. The approach resembles with the $r=4$ WENO interpolation (i.e., pointwise) algorithm described in [3] in terms of using a set of third degree polynomials. The $r=4$ WENO algorithm evaluates seventh order accurate cell interface values $q_{L ; R, i}$ over a seven-point stencil using four third degree polynomials. In contrast, our approach is to reconstruct an approximation to a first derivative of a function $q^{\prime}\left(x_{i}, t^{n}\right)$ [11] over a five-point stencil, using two third degree polynomials.

For this, we might consider using the same strategy in Section 2.2 in which the three second degree ENO polynomials in Eq. 25) are constructed over the five-point stencil $S=\cup_{\ell=1}^{3} S_{\ell}$. However, this setup will provide only a thirdorder accurate approximation $q_{C, i}^{\prime}$ to the exact derivative $q^{\prime}\left(x_{i}\right)$. To see this, we first observe that the smoothness indicators $\beta_{\ell}$ with this setup will be including only a single term,

$$
\beta_{\ell}=\Delta x^{3} \int_{I_{i}}\left[p_{\ell}^{\prime \prime}(x)\right]^{2} d x, \quad \ell=1,2,3 .
$$

10 Through a Taylor expansion analysis on Eq. 30 we see

$$
\beta_{\ell}=D(1+\mathcal{O}(\Delta x)),
$$


where $D=\left(q^{\prime \prime} \Delta x^{2}\right)^{2}$ is a nonzero quantity independent of $\ell$ but may depend on $\Delta x$, assuming $q^{\prime \prime} \neq 0$ on $S$. This results in a set of three nonlinear weights $\omega_{\ell}, \ell=1,2,3$, obtained either by Eq. 26) or Eq. 29, satisfying

$$
\omega_{\ell}=\gamma_{\ell}+\mathcal{O}(\Delta x)
$$

where the linear constant weights $\gamma_{\ell}$ are assumed to exist, when $q^{\prime}\left(x, t^{n}\right)$ is

215 smooth in $S$, such that

$$
q_{C, i}^{\prime}=\sum_{\ell=1}^{3} \gamma_{\ell} p_{\ell}^{\prime}\left(x_{i}\right)=q^{\prime}\left(x_{i}, t^{n}\right)+\mathcal{O}\left(\Delta x^{3}\right) .
$$

This finally implies the accuracy of $q_{C, i}^{\prime}$ is found out to be third-order,

$$
q_{C, i}^{\prime}=\sum_{\ell=1}^{3} \omega_{\ell} p_{\ell}^{\prime}\left(x_{i}\right)=q^{\prime}\left(x_{i}, t^{n}\right)+\mathcal{O}\left(\Delta x^{3}\right),
$$

because

$$
\begin{aligned}
\sum_{\ell=1}^{3} \omega_{\ell} p_{\ell}^{\prime}\left(x_{i}\right)-\sum_{\ell=1}^{3} \gamma_{\ell} p_{\ell}^{\prime}\left(x_{i}\right) & =\sum_{\ell=1}^{3}\left(\omega_{\ell}-\gamma_{\ell}\right)\left(p_{\ell}^{\prime}\left(x_{i}\right)-q^{\prime}\left(x_{i}, t^{n}\right)\right) \\
& =\sum_{\ell=1}^{3} \mathcal{O}(\Delta x) \mathcal{O}\left(\Delta x^{2}\right)=\mathcal{O}\left(\Delta x^{3}\right) .
\end{aligned}
$$

In the last equality, we used the fact that, for each $\ell, p_{\ell}^{\prime}(x)$ is only a first degree polynomial which is accurate up to second-order when approximating $q^{\prime}\left(x, t^{n}\right)$.

For this reason, we want a better strategy to obtain an approximation $q_{C, i}^{\prime}$ at least fourth-order accurate in order that the overall nominal accuracy of the 1D PCM scheme achieves at least fourth-order accurate in both space and time.

Step 1: PPM-Build. An alternate strategy for this goal therefore would be to use a set of two third degree polynomials $\phi_{ \pm}(x)$ over the same five-point stencil $S=\cup_{\ell=1}^{2} S_{\ell}$, where each polynomial is defined on $S_{1}=\left\{I_{i-2}, \ldots, I_{i+1}\right\}$ and $S_{2}=\left\{I_{i-1}, \ldots, I_{i+2}\right\}$. This can be designed using the two third degree polynomials, $\phi_{ \pm}(x)$, from the PPM algorithm [1],

$$
\phi_{ \pm}(x)=\sum_{k=0}^{3} a_{k}^{ \pm}\left(x-x_{i \pm 1 / 2}\right)^{k},
$$

where the coefficients are determined in such a way that $\phi_{ \pm}$preserves the volume average on each cell $I_{i}$. The explicit forms of $a_{k}^{ \pm}$are given in Appendix B. 
over the stencil $S=\cup_{\ell=1}^{2} S_{\ell}$, we use their first derivatives $\phi_{ \pm}^{\prime}$ to obtain a convex combination with two linear weights $\gamma_{-}$and $\gamma_{+}$,

$$
q_{C, i}^{\prime}=\gamma_{-} \phi_{-}^{\prime}\left(x_{i}\right)+\gamma_{+} \phi_{+}^{\prime}\left(x_{i}\right)
$$

The two linear weights are determined to obtain a fourth order accuracy in $q_{C, i}^{\prime}$,

$$
\gamma_{-}=\gamma_{+}=\frac{1}{2}
$$

We display more details in Appendix B.

Step 3: Nonlinear Weights. The smoothness indicators $\beta_{ \pm}$are now constructed using $\phi_{ \pm}(x)$ as

$$
\beta_{ \pm}=\sum_{s=2}^{3}\left(\Delta x^{2 s-1} \int_{I_{i}}\left[\frac{d^{s}}{d x^{s}} \phi_{ \pm}(x)\right]^{2} d x\right)
$$

It is to be noted that the summation begins from $s=2$ to measure their smoothness from the second derivative of $\phi_{ \pm}(x)$ because $q_{C, i}^{\prime}$ are our building blocks in this case. They can be written as

$$
\beta_{-}=4\left(a_{2}^{-}\right)^{2} \Delta x^{4}+12\left(a_{2}^{-}\right)\left(a_{3}^{-}\right) \Delta x^{5}+48\left(a_{3}^{-}\right)^{2} \Delta x^{6}
$$

240 and

$$
\beta_{+}=4\left(a_{2}^{+}\right)^{2} \Delta x^{4}-12\left(a_{2}^{+}\right)\left(a_{3}^{+}\right) \Delta x^{5}+48\left(a_{3}^{+}\right)^{2} \Delta x^{6}
$$

Upon conducting Taylor series expansion analysis on $\beta_{ \pm}$, we can see that

$$
\beta_{ \pm}=D(1+\mathcal{O}(\Delta x))
$$

where $D=\left(q^{\prime \prime} \Delta x^{2}\right)^{2}$ is a nonzero quantity independent of \pm but might depend on $\Delta x$, assuming $q^{\prime \prime} \neq 0$ on $S$. It is observed that unlike the smoothness indicators in Eqs. A.8, the new two smoothness indicators in Eqs. 40 and

(41) are no longer written as a sum of perfect squares. Recently, Balsara et al. [35. reformulated the smoothness indicators as a sum of perfect squares for $r=3$ to $r=9$ finite difference WENO schemes. This compact form helps their new 
finite difference adaptive WENO-AO schemes become computationally more efficient than the previous approach reported in [3].

The remaining procedure is to obtain the two nonlinear weights $\omega_{ \pm}$in the similar way done in the edge reconstructions in Eqs. (26) - 29):

- For WENO-JS:

$$
\omega_{ \pm}^{J S}=\frac{\tilde{\omega}_{ \pm}}{\tilde{\omega}_{-}+\tilde{\omega}_{+}}, \text {where } \tilde{\omega}_{ \pm}=\frac{\gamma_{ \pm}}{\left(\epsilon+\beta_{ \pm}\right)^{m}}
$$

- For WENO-M:

$$
\omega_{ \pm}^{M}=\frac{\tilde{\omega}_{ \pm}}{\tilde{\omega}_{-}+\tilde{\omega}_{+}}
$$

255

where

$$
\tilde{\omega}_{ \pm}=g\left(\omega_{ \pm}^{J S}\right)=\frac{\omega_{ \pm}^{J S}\left(\gamma_{ \pm}+\left(\gamma_{ \pm}\right)^{2}-3\left(\gamma_{ \pm}\right) \omega_{ \pm}^{J S}+\left(\omega_{ \pm}^{J S}\right)^{2}\right)}{\left(\gamma_{ \pm}\right)^{2}+\omega_{ \pm}^{J S}\left(1-2 \gamma_{ \pm}\right)}
$$

\section{- For WENO-Z:}

$$
\omega_{ \pm}^{Z}=\frac{\tilde{\omega}_{ \pm}}{\tilde{\omega}_{-}+\tilde{\omega}_{+}}, \text {where } \tilde{\omega}_{ \pm}=\gamma_{ \pm}\left(1+\left(\frac{\left|\beta_{+}-\beta_{-}\right|}{\epsilon+\beta_{ \pm}}\right)^{m}\right)
$$

The final representation of the approximation $q_{C, i}^{\prime}$ becomes

$$
q_{C, i}^{\prime}=\omega_{-} \phi_{-}^{\prime}\left(x_{i}\right)+\omega_{+} \phi_{+}^{\prime}\left(x_{i}\right) .
$$

Let us now verify that this approximation is fourth-order accurate after all, that 260 is,

$$
q_{C, i}^{\prime}=\omega_{-} \phi_{-}\left(x_{i}\right)+\omega_{+} \phi_{+}\left(x_{i}\right)=q^{\prime}\left(x_{i}, t^{n}\right)+\mathcal{O}\left(\Delta x^{4}\right) .
$$

Similarly as before, using Eqs. (42), (43), and (46), we can see that, with the help of the binomial series expansion,

$$
\omega_{ \pm}=\gamma_{ \pm}+\mathcal{O}(\Delta x)
$$


Therefore, the desired accuracy claimed in Eq. 48 is readily verified by repeating the similar relationship in Eq. (35):

$$
\begin{aligned}
\sum_{\ell=-,+} \omega_{\ell} \phi_{\ell}^{\prime}\left(x_{i}\right)-\sum_{\ell=-,+} \gamma_{\ell} \phi_{\ell}^{\prime}\left(x_{i}\right) & =\sum_{\ell=-,+}\left(\omega_{\ell}-\gamma_{\ell}\right)\left(\phi_{\ell}^{\prime}\left(x_{i}\right)-q^{\prime}\left(x_{i}, t^{n}\right)\right) \\
& =\sum_{\ell=-,+} \mathcal{O}(\Delta x) \mathcal{O}\left(\Delta x^{3}\right)=\mathcal{O}\left(\Delta x^{4}\right) .
\end{aligned}
$$

265 due to the improved third-order accuracy in calculating $\phi_{\ell}^{\prime}\left(x_{i}\right)-q^{\prime}\left(x_{i}, t^{n}\right)$. This is a result of using the third degree PPM polynomials $\phi_{ \pm}(x)$, with which $q^{\prime}\left(x, t^{n}\right)$ can be accurately approximated by the second degree polynomials $\phi_{ \pm}^{\prime}(x)$ up to third-order. order accurate Riemann states $q_{L ; R, i}$, and the fourth-order accurate derivative $q_{C, i}^{\prime}$ in space. The remaining task includes conducting a temporal updating step via tracing the characteristic lines using the piecewise cubic polynomials in Eq. 99. This step produces the Riemann states $\left(q_{L}, q_{R}\right)=\left(q_{R, i}^{n+1 / 2}, q_{L, i+1}^{n+1 / 2}\right)$

fourth-order accurate in time for 1D cases, and they are provided as the initial value problems for the Godunov fluxes at each interface $x_{i+1 / 2}$.

\section{The PCM Characteristic Tracing for Temporal Updates}

The PCM proceeds to the last step which advances the pointwise Riemann interface states at $t^{n}$

$$
q_{L ; R, i}=p_{i}\left(x_{i \pm 1 / 2}\right)
$$

where $p_{i}(x)$ is the piecewise cubic polynomial in Eq. (9), to the half-time updated predictor states

$$
q_{L ; R, i}^{n+1 / 2}
$$

by tracing characteristics. The idea is same as how the PPM characteristic tracing is performed [1], in which we seek a time averaged state. For instance, 
at the interface $x_{i+1 / 2}$, we consider

$$
q_{x+1 / 2}^{n+1 / 2}=\frac{1}{\Delta t} \int_{t^{n}}^{t^{n+1}} q\left(x_{i+1 / 2}, t\right) d t
$$

The initial condition at $t^{n}$ of a Generalized Riemann Problem (GRP) is given as

$$
q\left(x_{i+1 / 2}, t^{n}\right)= \begin{cases}p_{i}\left(x_{i+1 / 2}\right), & x \in I_{i} \\ p_{i+1}\left(x_{i+1 / 2}\right), & x \in I_{i+1} .\end{cases}
$$

Given a linear characteristic equation as in Eq. (8), and for $t>t^{n}$ we then have

$$
q\left(x_{i+1 / 2}, t\right)= \begin{cases}p_{i}\left(x_{i+1 / 2}-\lambda_{i}\left(t-t^{n}\right)\right), & x \in I_{i}, \quad \lambda_{i}>0, \\ p_{i+1}\left(x_{i+1 / 2}-\lambda_{i+1}\left(t-t^{n}\right)\right), & x \in I_{i+1}, \quad \lambda_{i+1}<0 .\end{cases}
$$

Here $\xi(t)=x_{i+1 / 2}-\lambda\left(t-t^{n}\right)$ is a characteristic line for an eigenvalue $\lambda$, assuming $t-t^{n}<\Delta t$. In this approach we do not solve the Generalized Riemann Problem directly. Rather we use a trapezium rule approximation in time to the integral in Eq. 53 , where $q\left(x_{i+1 / 2}, t\right)$ the solution of the GRP along the $t$-axis at the interface $x_{i+1 / 2}$ in the $t-x$ plane. Although this approach is very simple and can be considered to be very similar to the second order characteristic tracing algorithms of PLM and PPM [36, 1] we see there is a drawback in general. As it stands, the state $q\left(x_{i+1 / 2}, t\right)$ constructed using the linear characteristic line $\xi(t)$ in Eq. 55 is not fully justified in the cases of the Generalized Riemann problem where the characteristics are curved lines instead of straight lines in the $x$ - $t$ plane. On the other hand, it has been shown by Qian et al. 22] that a framework of high-order GRP methods can be formulated for second and third order accuracy by using a linear and quadratic GRP solvers respectively. Their approaches, relying on new treatments of resolving curved rarefaction and discontinuity waves, illustrate that the use of linear characteristics is not entirely supported in the third and higher order cases, especially for the system of cases.

Regardless, our simple characteristic tracing strategy has shown that a fast rate of convergence can be achieved for $\Delta t \sim \Delta x$, which is now described below for the case with $x \in I_{i}$ with $\lambda_{i}>0$ first. Using $\uparrow$ to denote the state from the 
left of $x_{i+1 / 2}$,

$$
\begin{aligned}
q_{R, i}^{n+1 / 2}=q_{x+1 / 2, \uparrow}^{n+1 / 2} & =\frac{1}{\Delta t} \int_{t^{n}}^{t^{n+1}} q\left(x_{i+1 / 2}, t\right) d t \\
& =\frac{1}{\lambda_{i} \Delta t} \int_{x_{i+1 / 2-\lambda_{i} \Delta t}}^{x_{i+1 / 2}} p_{i}(x) d x
\end{aligned}
$$

310

$$
\begin{aligned}
q_{R, i}^{n+1 / 2} & =q_{x+1 / 2, \uparrow}^{n+1 / 2} \\
& =c_{0}+\frac{c_{1}}{2}\left(1-\frac{\lambda_{i} \Delta t}{\Delta x}\right) \Delta x+\frac{c_{2}}{4}\left(1-2 \frac{\lambda_{i} \Delta t}{\Delta x}+\frac{4}{3}\left(\frac{\lambda_{i} \Delta t}{\Delta x}\right)^{2}\right) \Delta x^{2} \\
& +\frac{c_{3}}{8}\left(1-3 \frac{\lambda_{i} \Delta t}{\Delta x}+4\left(\frac{\lambda_{i} \Delta t}{\Delta x}\right)^{2}-2\left(\frac{\lambda_{i} \Delta t}{\Delta x}\right)^{3}\right) \Delta x^{3}
\end{aligned}
$$

The case for $x \in I_{i+1}$ with $\lambda_{i+1}<0$ can be obtained similarly,

$$
\begin{aligned}
q_{L, i+1}^{n+1 / 2} & =q_{x+1 / 2, \downarrow}^{n+1 / 2} \\
& =c_{0}+\frac{c_{1}}{2}\left(-1-\frac{\lambda_{i+1} \Delta t}{\Delta x}\right) \Delta x+\frac{c_{2}}{4}\left(1+2 \frac{\lambda_{i+1} \Delta t}{\Delta x}+\frac{4}{3}\left(\frac{\lambda_{i+1} \Delta t}{\Delta x}\right)^{2}\right) \Delta x^{2} \\
& +\frac{c_{3}}{8}\left(-1-3 \frac{\lambda_{i+1} \Delta t}{\Delta x}-4\left(\frac{\lambda_{i+1} \Delta t}{\Delta x}\right)^{2}-2\left(\frac{\lambda_{i+1} \Delta t}{\Delta x}\right)^{3}\right) \Delta x^{3}
\end{aligned}
$$

In the general case of a system of Euler equations, the above treatment is to be extended to include multiple characteristic waves correspondingly depending on the sign of each $k$-th eigenvalue $\lambda_{i}^{(k)}$. This gives us, for the two predictor 320 states $\mathbf{V}_{L ; R, i}^{n+1 / 2}$ on each cell $I_{i}$ in primitive form,

$$
\begin{aligned}
& \mathbf{V}_{R, i}^{n+1 / 2}=\mathbf{C}_{0}+\frac{1}{2} \sum_{k ; \lambda_{i}^{(k)}>0}\left(1-\frac{\lambda_{i}^{(k)} \Delta t}{\Delta x}\right) r^{(k)} \Delta \mathbf{C}_{1}^{(k)} \\
& +\frac{1}{4} \sum_{k ; \lambda_{i}^{(k)}>0}\left(1-2 \frac{\lambda_{i}^{(k)} \Delta t}{\Delta x}+\frac{4}{3}\left(\frac{\lambda_{i}^{(k)} \Delta t}{\Delta x}\right)^{2}\right) r^{(k)} \Delta \mathbf{C}_{2}^{(k)},
\end{aligned}
$$




$$
+\frac{1}{8} \sum_{k ; \lambda_{i}^{(k)}>0}\left(1-3 \frac{\lambda_{i}^{(k)} \Delta t}{\Delta x}+4\left(\frac{\lambda_{i}^{(k)} \Delta t}{\Delta x}\right)^{2}-2\left(\frac{\lambda_{i}^{(k)} \Delta t}{\Delta x}\right)^{3}\right) r^{(k)} \Delta \mathbf{C}_{3}^{(k)}
$$

and

$$
\begin{aligned}
& \mathbf{V}_{L, i}^{n+1 / 2}=\mathbf{C}_{0}+\frac{1}{2} \sum_{k ; \lambda_{i}^{(k)}<0}\left(-1-\frac{\lambda_{i}^{(k)} \Delta t}{\Delta x}\right) r^{(k)} \Delta \mathbf{C}_{1}^{(k)} \\
& +\frac{1}{4} \sum_{k ; \lambda_{i}^{(k)}<0}\left(1+2 \frac{\lambda_{i}^{(k)} \Delta t}{\Delta x}+\frac{4}{3}\left(\frac{\lambda_{i}^{(k)} \Delta t}{\Delta x}\right)^{2}\right) r^{(k)} \Delta \mathbf{C}_{2}^{(k)}, \\
& +\frac{1}{8} \sum_{k ; \lambda_{i}^{(k)}<0}\left(-1-3 \frac{\lambda_{i}^{(k)} \Delta t}{\Delta x}-4\left(\frac{\lambda_{i}^{(k)} \Delta t}{\Delta x}\right)^{2}-2\left(\frac{\lambda_{i}^{(k)} \Delta t}{\Delta x}\right)^{3}\right) r^{(k)} \Delta \mathbf{C}_{3}^{(k)} .
\end{aligned}
$$

Those new notations introduced in Eqs. 60 and (61) represent the $k$-th right eigenvector $r^{(k)}, k=1,2,3$, which is the $k$-th column vector of the $3 \times 3$ matrix $\mathbf{R}$ evaluated at $I_{i}$,

$$
\mathbf{R}=\left[r^{(1)}\left|r^{(2)}\right| r^{(3)}\right]
$$

325 and the $k$-th characteristic variable vector $\Delta \mathbf{C}_{m}^{(k)}$ given as

$$
\Delta \mathbf{C}_{m}^{(k)}=\Delta x^{m} \ell^{(k)} \cdot \mathbf{C}_{m}
$$

where, for $m=0, \ldots, 3$,

$$
\mathbf{C}_{m}=\left[c_{m}^{(1)}\left|c_{m}^{(2)}\right| c_{m}^{(3)}\right]^{T}
$$

in which $c_{m}^{(k)}$ is the $m$-th coefficient in Eqs. 21,24 of the piecewise cubic polynomial in Eq. (9) applied to each of the $k$-th characteristic variable $\bar{q}_{i}$.

It is worth mentioning that, unlike the characteristic tracing of PPM (see 330 [1]), the PCM scheme does not necessarily require any extra monotonicity enforcements on $\mathbf{V}_{L ; R, i}^{n+1 / 2}$. This is mainly because the PCM reconstruction makes full use of the WENO reconstruction algorithms, providing non-oscillatory approximations to $q_{L ; R, i}$ and $q_{C, i}^{\prime}$ by design. 


\section{Final Update Step in 1D}

335 $\mathcal{R} \mathcal{P}\left(\mathbf{U}_{L}, \mathbf{U}_{R}\right)=\mathcal{R} \mathcal{P}\left(\mathbf{U}_{R, i}^{n+1 / 2}, \mathbf{U}_{L, i+1}^{n+1 / 2}\right)$ as corrector, where $\mathcal{R} \mathcal{P}$ implies a solution of the Riemann problem. Note that the Riemann states in conservative variables $\mathbf{U}_{R, i}^{n+1 / 2}, \mathbf{U}_{L, i+1}^{n+1 / 2}$ are obtained either by conversions from $\mathbf{V}_{R, i}^{n+1 / 2}, \mathbf{V}_{L, i+1}^{n+1 / 2}$ in Eq. (60) and Eq. (61), or projecting the characteristic variables directly to needs to be processed using high-order approximation [14, in particular for multidimensonal problems, while such a high-order conversion is not required in $1 \mathrm{D}$. In this regards the latter could be a better choice in multi spatial dimensions, because there is no need for any high-order conversion from the primitive Riemann states to the conservative Riemann states, knowing the fact that the conservative states variables are the type of inputs for the Riemann problems.

\section{Multidimensional Extension of the 1D PCM Scheme}

Our primary purpose in the current paper is to focus on laying down the key algorithmic components of PCM in 1D. As described, the 1D PCM algorithm is formally fifth-order in space and fourth-order in time. Our test problems of one-dimensional smooth flows in Section 6.1.1 show that the algorithm delivers nominally a fifth-order accurate convergence rate, particularly with smaller $L_{1}$ errors than WENO-JS with RK4.

Although possible, extending such a high-order 1D algorithm to multiple spatial dimensions in a way to preserve the same order of convergence in $1 \mathrm{D}$ is an attentive task that requires some extra cares and attentions [11, 12, 13, 14] in the finite volume formulation. On the other hand, one of the simplest and easiest directionally unsplit multidimensional extensions that has been widely adopted in many algorithmic choices $[37,38,39,40,41,42,43,44,45,46,47$, is to use the so-called dimension-by-dimension formalism [12. In this approach, a finite volume scheme adopts a $1 \mathrm{D}$ reconstruction procedure to obtain high order accurate edge values at cell faces, use them to approximate fluxes at a 
single barycentric point on each cell face, and update the conserved variables using those fluxes in a directionally unsplit way. However, the order of convergence from the resulting multidimensional extension is limited to be at most second-order due to the lack of accuracies that may arise in a couple of places in code implementations: (i) mis-using averaged quantities in place of pointwise quantities for Riemann states, (ii) using low-order approximations in converting between primitive and conservative variables, (iii) and applying low-order quadrature rules in flux function estimations [11, 12, 13, 14. In our case, it takes more coding efforts, practically because the multidimensional PCM results we demonstrate in this paper have been obtained by integrating the PCM algorithm in the FLASH code framework 41, 42, 48; hence carrying out the above-mentioned code changes in a large code such as FLASH requires extra 375 efforts that are not the main points of the current paper. We leave such a highorder, multidimensional extension in our future work, and instead, we adopt the simple dimension-by-dimension formalism for our multidimensional extension of the 1D PCM algorithm.

Additionally, for our choice of multidimensional extension we use the computationally efficient unsplit corner transport upwind (CTU) formulation in FLASH [43, 44, which requires smaller number of Riemann solves in both 2D and 3D than the conventional CTU approaches [49, 50, 51, while achieving the maximum Courant condition of $\mathrm{CFL} \approx 1$ [43, 44].

\section{Results}

In this section we present numerical results of PCM in 1D, 2D and 3D for hydrodynamics and magnetohydrodynamics. The PCM results are compared with numerical solutions of other popular choices of reconstruction schemes including the second-order PLM [36, the third-order PPM [1] and the fifthorder WENO methods [2, 27, 28. As mentioned, the second-order accurate dimension-by-dimension approach has been adopted to extend all of the above baseline 1D reconstruction algorithms to multiple spatial dimensions. As this is 
the case, for multidimensional problems we have chosen the predictor-corrector type of characteristic tracing methods (charTr) for PLM, PPM, and WENO ${ }^{\dagger}$ not to mention PCM by design. In 1D problems, however, we treat WENO differently and integrate its spatial reconstruction with RK4 in consideration of fully demonstrating its orders of accuracy due from both space (i.e., $\mathcal{O}\left(\Delta x^{5}\right)$ ) and time (i.e., $\mathcal{O}\left(\Delta t^{4}\right)$ ). It should also be noted that the orders of WENO + RK4 in 1D are to be well comparable to those of PCM. Hence the choice provides a set of good informative comparisons between PCM and WENO + RK4 in particular, in which we will illuminate the advantages of PCM. In what follows, unless otherwise mentioned, we set the WENO-JS approach in Eq. 43p as the default choice for $q_{C, i}^{\prime}$ in our PCM results. This default setting will be referred to as PCM-JS (or simply PCM), while the choice with the WENO-Z approach in Eq. 46) will be referred to as PCM-Z.

6.1. $1 D$ Tests

\subsubsection{D Convergence and Performance Tests}

Gaussian and Sinusoidal Wave Advections. In our first test we consider two configurations of 1D passive advection of smooth flows, involving initial density profiles of Gaussian and sinusoidal waves. We initialize the both problems on a computational box on $[0,1]$ with periodic boundary conditions. The initial density profile of the Gaussian advection is defined by $\rho(x)=1+e^{-100\left(x-x_{0}\right)^{2}}$, with $x_{0}=0.5$, whereas for the sinusoidal advection the density is initialized by $\rho(x)=1.5-0.5 \sin (2 \pi x)$. In both cases, we set constant velocity, $u=1$, and pressure, $P=1 / \gamma$, and the specific heat ratio, $\gamma=5 / 3$.

The resulting profiles are propagated for one period through the boundaries, reaching $t=1$. At this point, both profiles return to its initial positions at which we conduct the $L_{1}$ error convergence tests compared with the initial conditions

\footnotetext{
${ }^{\dagger}$ To implement a characteristic tracing for WENO we first reconstruct the fifth-order Riemann states, $q_{S, i}^{\text {weno }, n}, S=L, R$, using WENO. They are then temporally evolved by $\Delta t / 2$ to get $q_{S, i}^{\text {weno, } n+1 / 2}$ by integrating over each corresponding domain of dependence the piecewise parabolic polynomials defined by $q_{L, i}^{w e n o, n}, q_{R, i}^{w e n o, n}$ and $\bar{q}_{i}$ [4].
} 


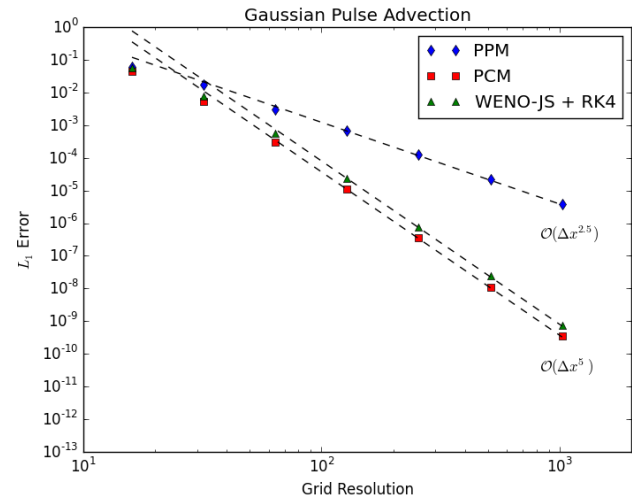

(a)

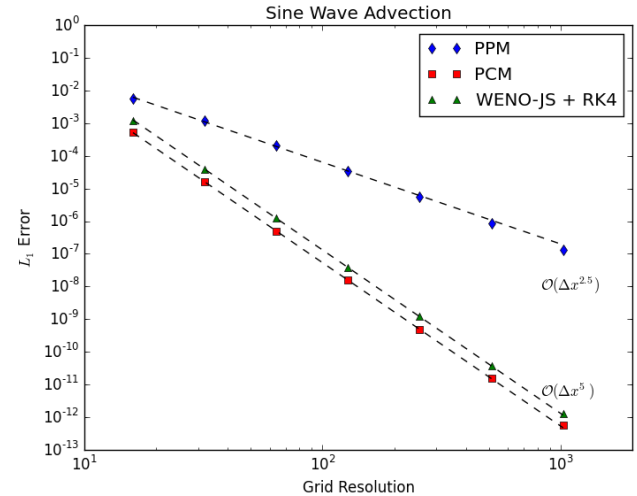

(b)

Figure 1: Convergence test of (a) 1D Gaussian pulse advection, and (b) 1D sinusoidal wave advection.

on the grid resolutions of $N_{x}=16,32,64,128,256,512$ and 1024 . Since the nature of the problem is a pure advection in both, any deformation of the initial profile is due to either phase errors or numerical diffusion. For stability we use a fixed Courant number, $C_{\mathrm{cfl}}=0.8$ for both tests. We choose the HLLC Riemann solver [52] in all cases.

The results of this study are shown in Fig. 1. From these numerical experiments, the PCM reconstruction shows the fifth-order convergence rates in both tests. Although both PCM and WENO-JS + RK4 demonstrate the same fifthorder of convergence rate, the $L_{1}$ errors of PCM are more than twice smaller than those of WENO-JS + RK4. The solutions of PPM converge with the rate of 2.5 which is the slowest among the three. Parameter choices for the PPM runs include the use of the MC slope limiter applied to characteristic variables, no flattening, no contact discontinuity steepening, and no artificial viscosity (this setting for PPM remains the same in what follows).

In Table 1 we compare the relative performance speedups of PCM, PPM and WENO-JS + RK4, all testing the Gaussian and sinusoidal advection problems. We can clearly see that there is a big performance advantage in PCM over WENO-JS + RK4 in delivering the target fifth-order accuracy. The major 


\begin{tabular}{||l|c||}
\hline Scheme & Speedup \\
\hline PPM & 0.65 \\
PCM & 1.00 \\
WENO-JS + RK4 & 1.71 \\
\hline
\end{tabular}

Table 1: Relative speedup of the PPM and WENO schemes compared to the PCM scheme for the 1D Gaussian and sine advection problems. The comparisons have been obtained from a serial calculation on a single CPU.

gain in PCM lies in its predictor-corrector type of characteristic tracing which affords not only the accuracy but also the computational efficiency. Such a relative computational efficiency of PCM in 1D is expected to grow much larger in multidimensional problems, considering that there have to be added algorithmic complexities in achieving high-order accurate solutions in multidimensional finite volume reconstruction [11, 12, 13, 14, from the perspectives of balancing optimal numerical stability and accuracy. We will report our strategies of multidimensional extension of PCM in our future work.

\subsubsection{D Discontinuous Tests}

In this section we test PCM on a series of well-benchmarked shock-tube problems of one dimensional hydrodynamics and MHD that involve discontinuities and shocks. As all the tests here have already been well discussed in various literatures, we will describe their setups only briefly and put our emphasis more on discussing the code performance of PCM. Readers are encouraged to refer to the cited references in the texts for more detailed descriptions on each setup.

(a) Sod Shock Tube. The Sod's problem [53] has been one of the most widely chosen popular tests in 1D to assess a code's capability to handle shocks and contact discontinuities. The initial condition is consist of the left and the right states given as

$$
(\rho, u, p)= \begin{cases}(1,0,1) & x<0.5 \\ (0.125,0,0.1) & x>0.5\end{cases}
$$




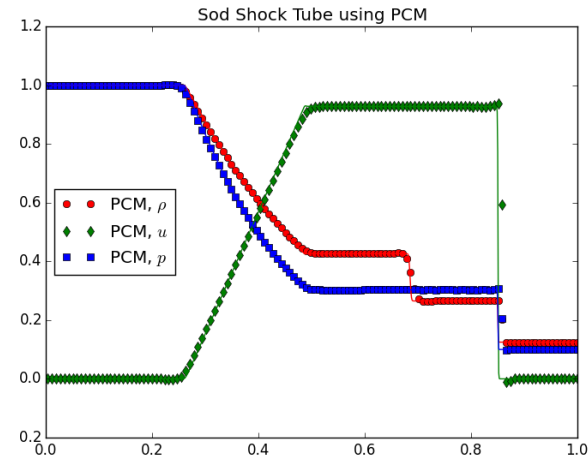

(a)

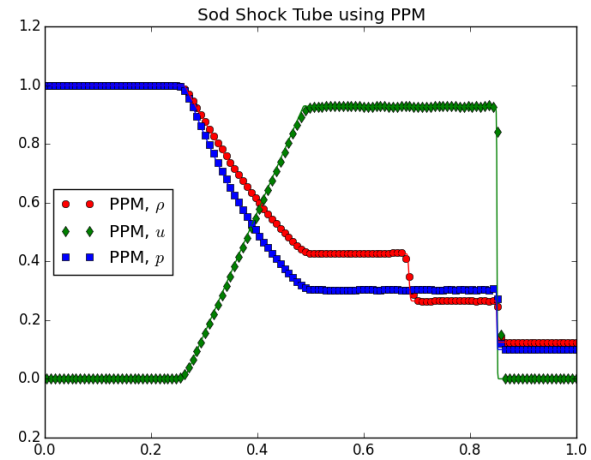

(b)

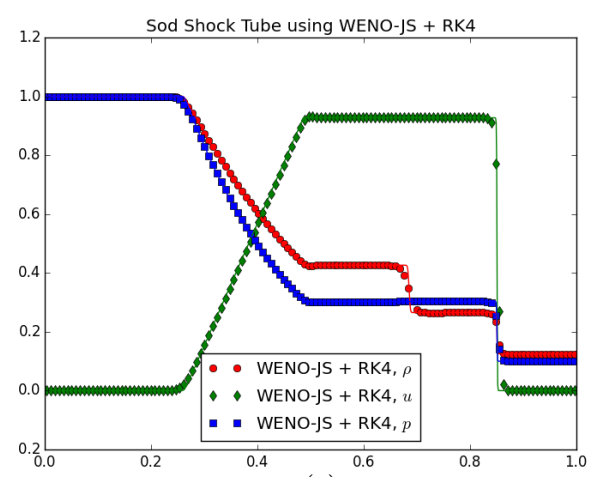

(c)

Figure 2: The Sod shock tube problem at $t=0.2$. (a) PMC, (b) PPM with MC slope limiter, and (c) WENO-JS + RK4. 
All methods were solved using the Roe Riemann solver, with $C_{\text {cfl }}=0.8$. It is evident in Fig. 3(b) that the PCM solution exhibits the least diffusive solution among the tested methods, producing a very-high order accurate solution that is more quickly approaching to the high resolution reference solution. 


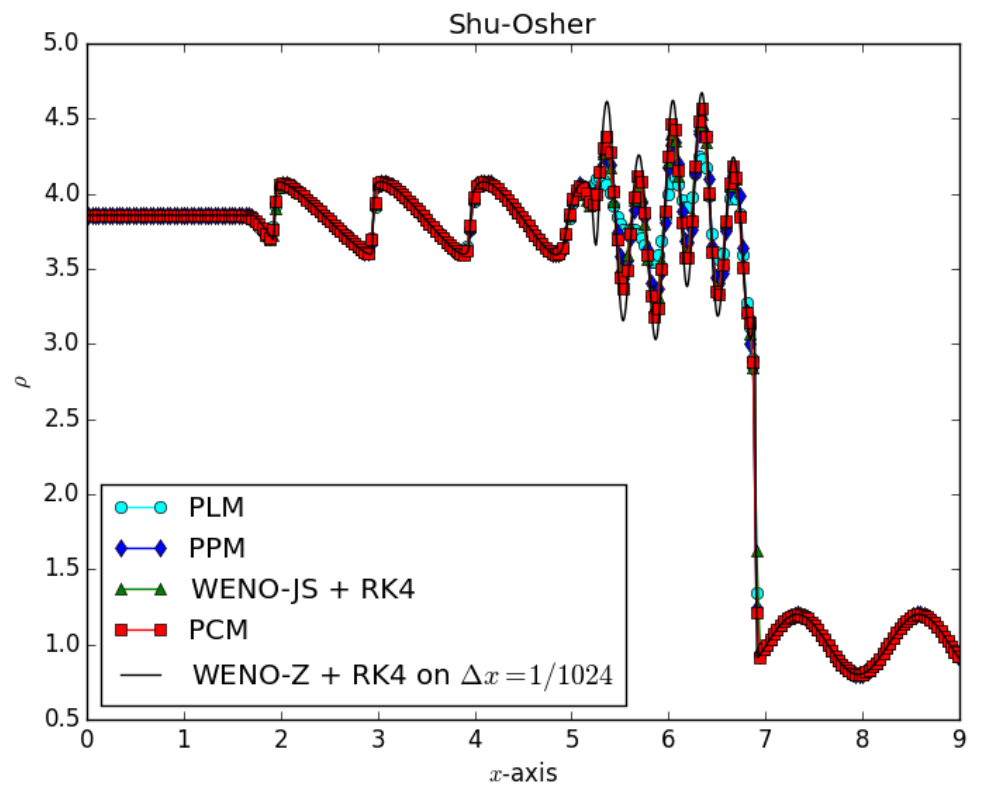

(a)

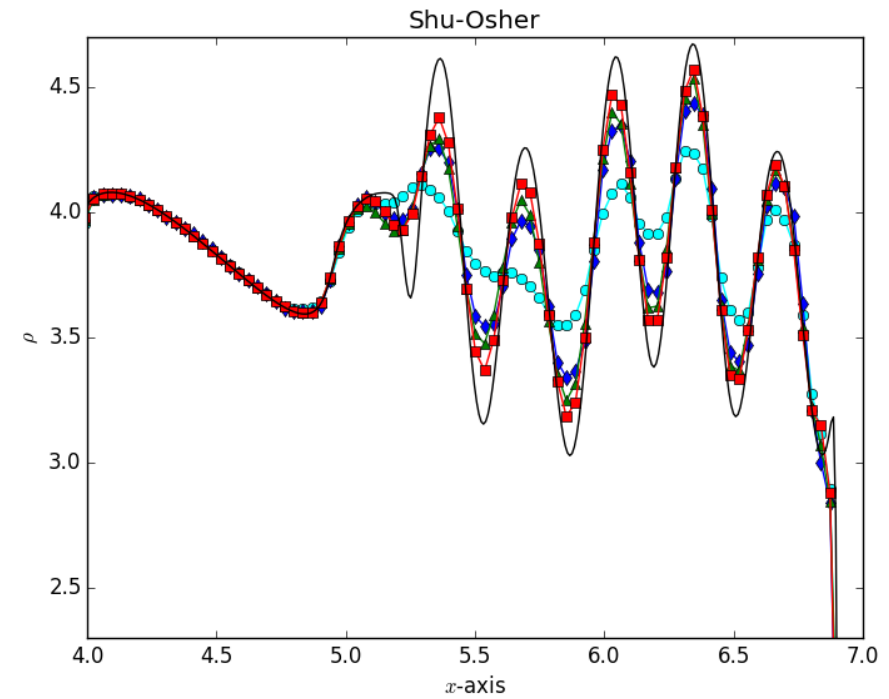

(b)

Figure 3: The Shu-Osher Riemann problem at $t=1.8$. (a) All four reconstruction schemes on $N_{x}=256$ are compared with WENO-JS + RK4 on $N_{x}=1024$. For PPM, the MC slope limiter is used. (b) A close-up view to demonstrate the schemes' numerical diffusivity. 


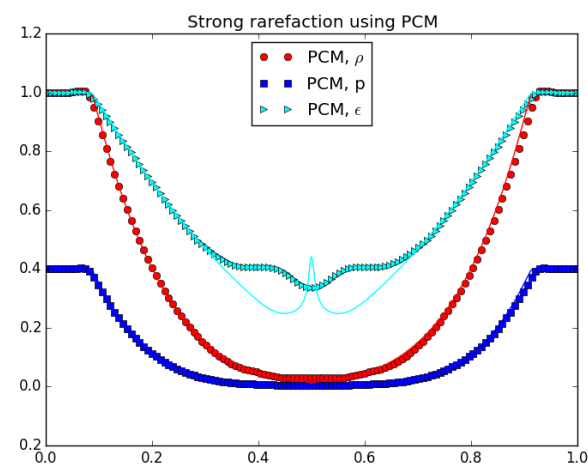

(a)

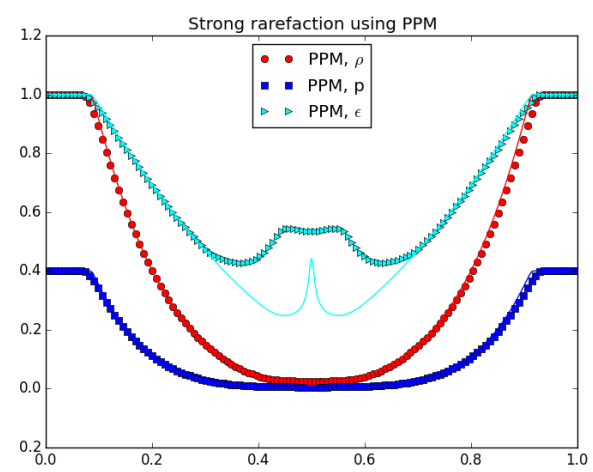

(b)

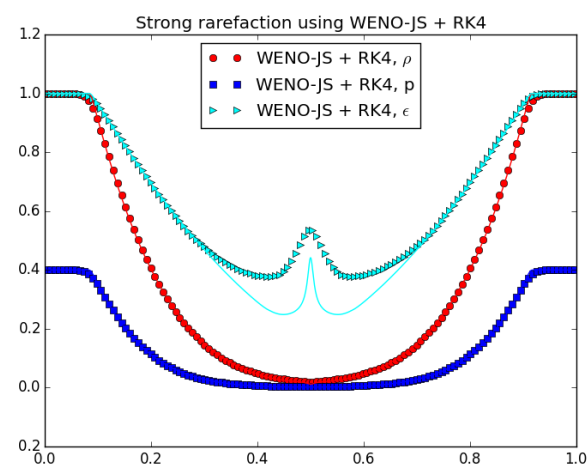

(c)

Figure 4: The Einfeldt strong rarefaction test at $t=0.15$. All tests used the HLLC Riemann solver on $N_{x}=128$ with $C_{\mathrm{cfl}}=0.8$. The solid curves represent the reference solution computed using WENO-JS + RK4 on $N_{x}=1024$.

(c) The Einfeldt Strong Rarefaction Test. First described by Einfeldt et al. [56] the main test point in this problem is to see how satisfactorily a code can compute physical variables, $p, u, \rho, \epsilon$, etc. in the low density region. Among the variables the internal energy $\epsilon=p /(\rho(\gamma-1))$, where $\gamma=1.4$, is the hardest to get it right due to the ratio of the pressure and density that are both close to zero. The ratio of the two small quantities will amplify any small errors in each, hence making the error in $\epsilon$ appear to be the largest in general [10].

The large errors in $\epsilon$ are indeed observed in Fig. 4(b) Fig. 4(c) in that the error is the largest at or around $x=0.5$ in the presence of sudden increase of its peak values. On the contrary, the internal energy computed using PCM shown 
in Fig. 4(a) behaves in a uniquely different way such that the value continues to drop when approaching $x=0.5$. From this viewpoint, and with the help of the exact solution available in [10, it's fair to say that the PCM solution in Fig. 4 (a) appears to predict the internal energy most accurately.

(d) Two-Blast. This problem was introduced by Woodward and Colella [57] and was designed to test a code performance particularly on interactions of strong shocks and discontinuities. We follow the original setup to test PCM, and compare its solution with those of PPM and WENO-JS + RK4, using 128 grid points to resolve the domain $[0,1]$. In Fig. 5 the three density profiles at $t=0.038$ are plotted against the high-resolution solution of WENO-JS + RK4 on 1024 grid points. Overall, all methods we tested here produce an acceptable quality of solutions as illustrated in Fig. 5(a). Note however that, among the three methods, the PCM solution in Fig. 5(b) demonstrates the highest peak heights, following more closely the high-resolution solutions. As reported in [40] we see that all methods also smear out the contact discontinuity at $x \approx 0.6$ pretty much the same amount.

(e) Brio-Wu MHD Shock Tube. An MHD version of the Sod's shock tube problem was first studied by Brio and $\mathrm{Wu}[33$, and it has become a must-to-do test for MHD codes. Since then, the problem has revealed a couple of interesting findings including not only the discovery of the compound wave [33, but also the existence of non-unique solutions [58, 59]. More recently, Lee 60] realized that there are unphysical numerical oscillations in using PPM and studied an approach to suppress the level of oscillations based on the upwind slope limiter. The presence of such oscillations in PPM has been also briefly reported in [40. The study reported in 60 shows that the origin of the oscillations arise from the numerical nature of a slowly moving shock as a function of the magnetic strength of tangential component. The slowly moving shock was first identified in [57, and the oscillatory behaviors have been studied by many researchers for more than 30 years, yet there is no ultimate resolution [61, 62, 63, 64, 65, 66. 


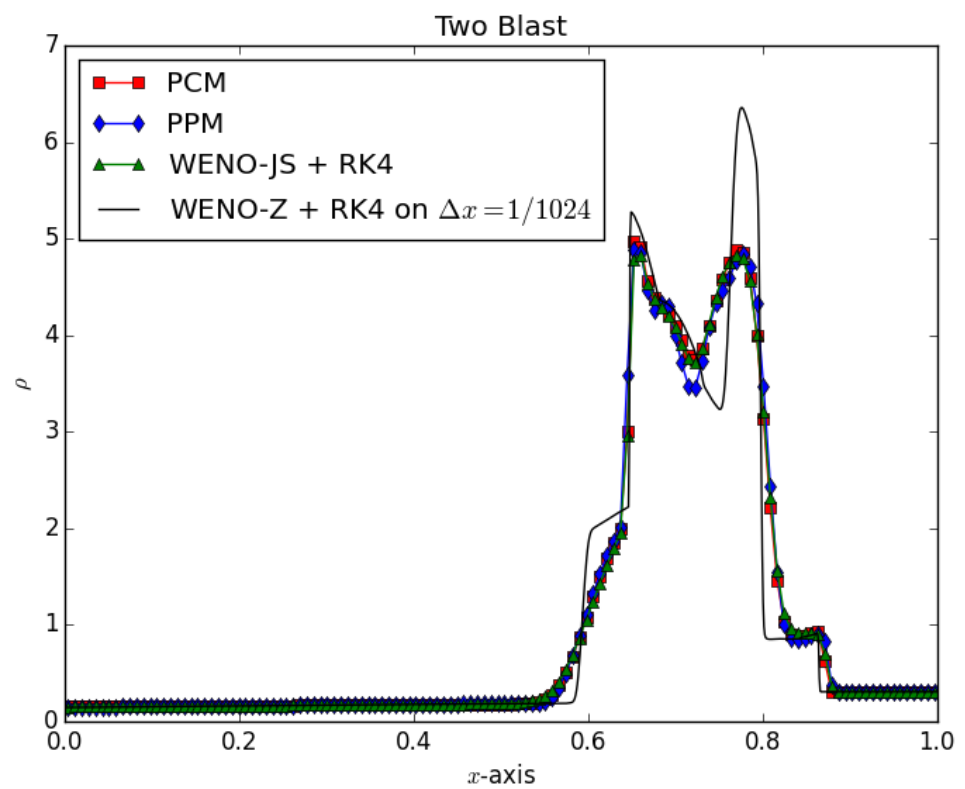

(a)

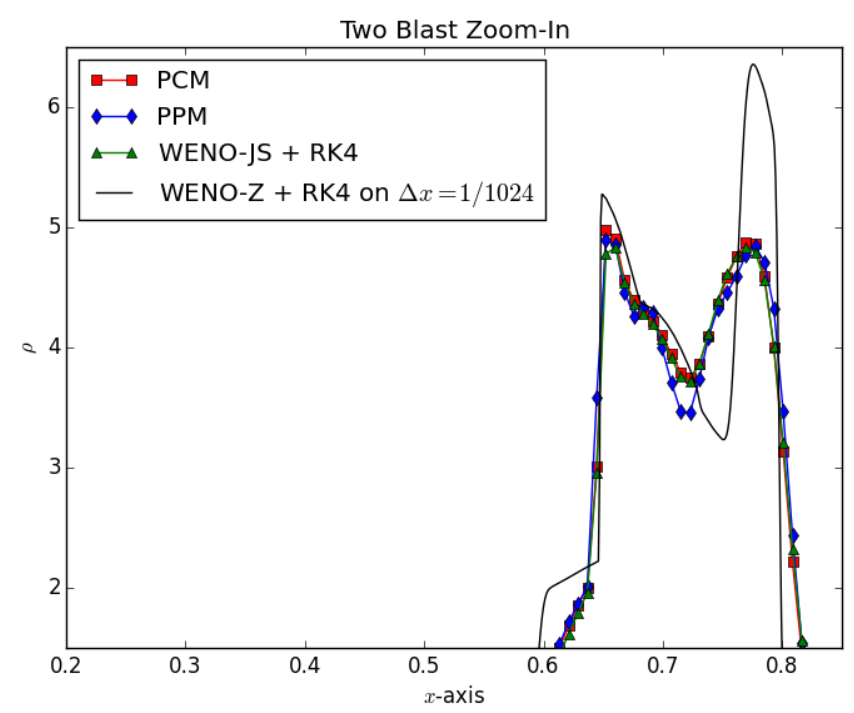

(b)

Figure 5: Two blast problem at $t=0.038$. (a) All three reconstruction schemes on $N_{x}=128$ are compared with WENO-JS + RK4 on $N_{x}=1024$. For PPM, the MC slope limiter is used. (b) A close-up view to demonstrate the schemes' numerical accuracy. In all tests we used the HLLC Riemann solver with $C_{\text {cfl }}=0.8$. 


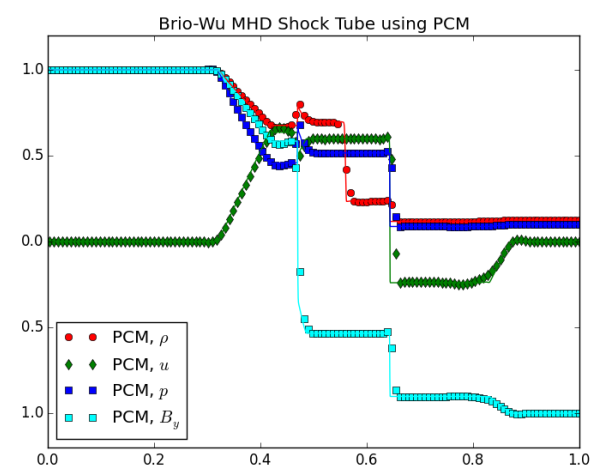

(a)

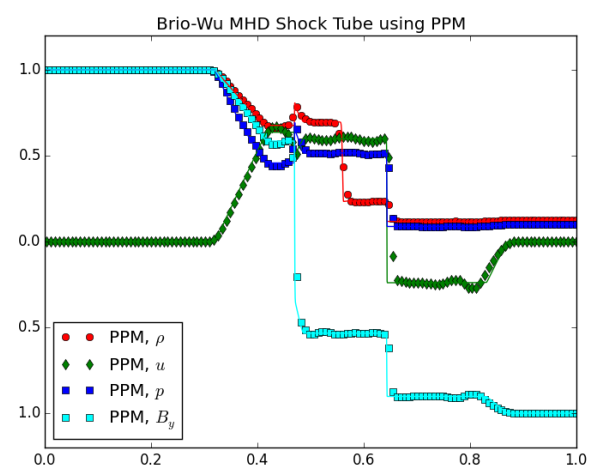

(b)

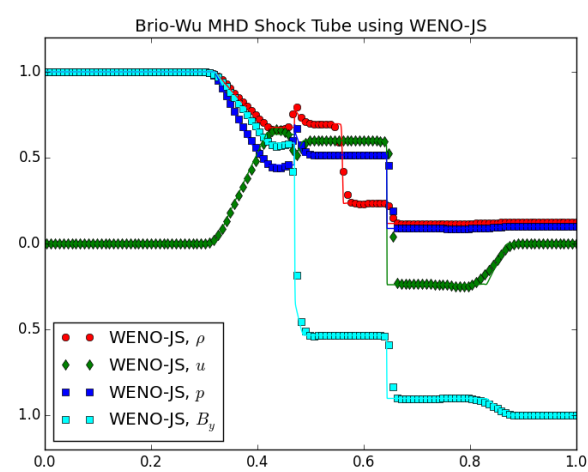

(c)

Figure 6: The Brio-Wu MHD shock tube problem. The Roe Riemann solver is consistently used for all methods. The test problems are computed on $N_{x}=128$, while the high-resolution reference solution is obtained using PLM on $N_{x}=2048$ with the MC slope limiter. The same slope limiter was used in PPM too. The Courant number is fixed as 0.8 in all runs.

In this test, as just mentioned, there are observable numerical oscillations found in all methods, PPM, PCM and WENO-JS + charTr. The results in Fig. 6 show that the oscillations are the largest in PPM, consistent with the findings in [60, and there are less amount in PCM and WENO-JS + charTr. The PPM solutions are suffering from significant amount of spurious oscillations in all four variables, $\rho, u, p$ and $B_{y}$, as shown in Fig. 6(b). Such behaviors are less significant in PCM and WENO-JS + charTr, respectively illustrated in Fig. 6(a) and Fig. 6(c), in that, the oscillations in $\rho, p, B_{y}$ are much more controlled now, while the most outstanding oscillations are found in $u$ near $x \approx 0.8$. It is worth 
mentioning that the oscillatory behaviors remain to be consistent regardless of the choice of Riemann solvers such as HLL [67, HLLC [68, HLLD [69], or Roe 54] (tested here).

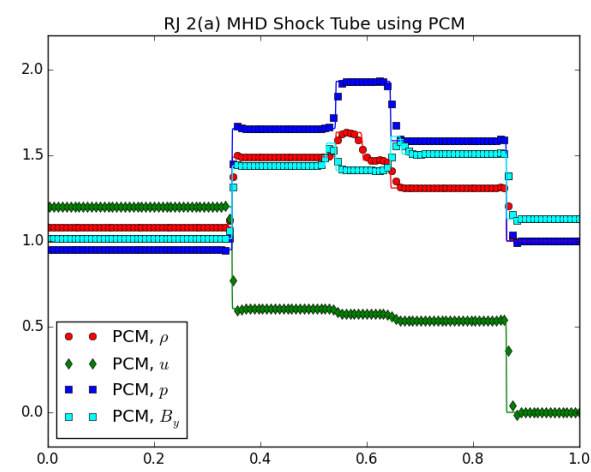

(a)

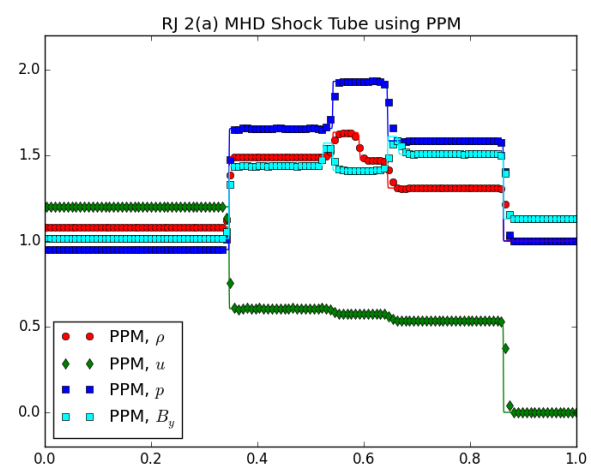

(b)

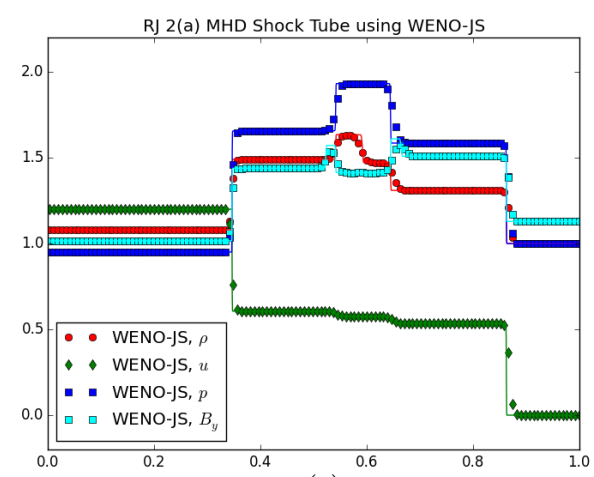

(c)

Figure 7: The RJ2a MHD shock tube problem. All runs used the HLLD Riemann solver, computed on $N_{x}=128$ with $C_{\text {cfl }}=0.8$. The reference solution was obtained using PLM on $N_{x}=2048$. The PLM and PPM methods used the MC slope limiter.

(f) RJ2a MHD Shock Tube. Ryu et al. [70] studied a class of one dimensional MHD shock tube problems that are informative to run as a code verification test. We have chosen one of their setups, introduced in their figure 2a. In what follows the problem is referred to be as the RJ2a test. The viewpoint of this test is to monitor if all three dimensional MHD waves are successfully captured. We see that in Fig. 7 all structures of left- and right-going fast shocks, leftand right-going slow shocks, and a contact discontinuity are well captured in all 


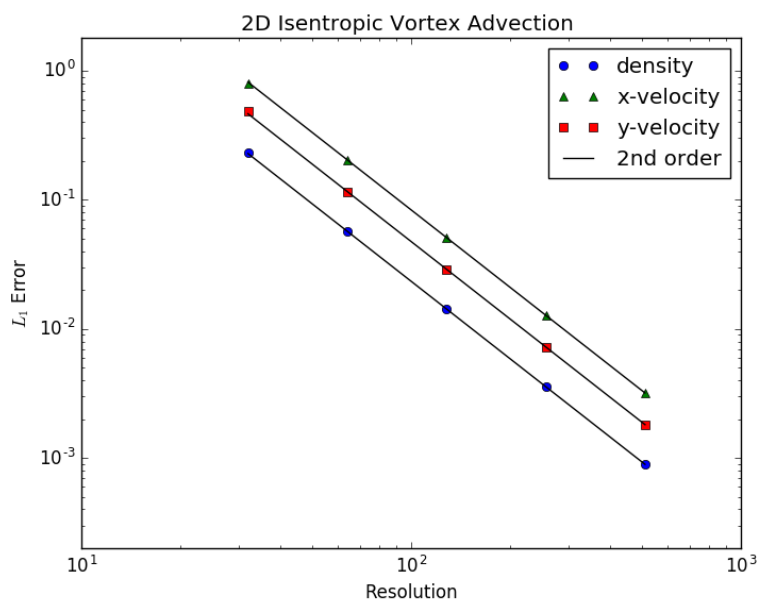

Figure 8: Convergence test of the 2D isentropic hydrodynamical vortex advection problem. The errors in $\rho, u$, and $v$ are calculated in $L_{1}$ sense against the initial conditions. The tested PCM solutions are solved on $N_{x} \times N_{y}$, where $N_{x}=N_{y}=32,64,128,256$ and 512. All runs reached to $t=10$ using the HLLC Riemann solver with $C_{\mathrm{cfl}}=0.8$.

methods tested, including PCM.

\section{2. $2 D$ Tests}

We present two dimensional tests of hydrodynamics and MHD in this section. All test cases are computed using the second-order dimension-by-dimension extension of the baseline 1D algorithms, including PCM.

\subsubsection{D Convergence Test of the Isentropic Vortex Advection - Hydro case}

The first 2D test problem, considered in [71, 3], consists of the advection of an isentropic vortex along the diagonal of a cartesian computational box. The dynamics of the problem allows to quantify a code's dissipative properties and the correct discretization balance of multidimensional terms through monitoring the preservation of the initial circular shape of the vortex. At $t=10$ the vortex finishes one periodic advection over the domain and returns to the initial position, where we can measure the solution accuracy against the initial condition. As such we have chosen this problem particularly to access the PCM's order of 


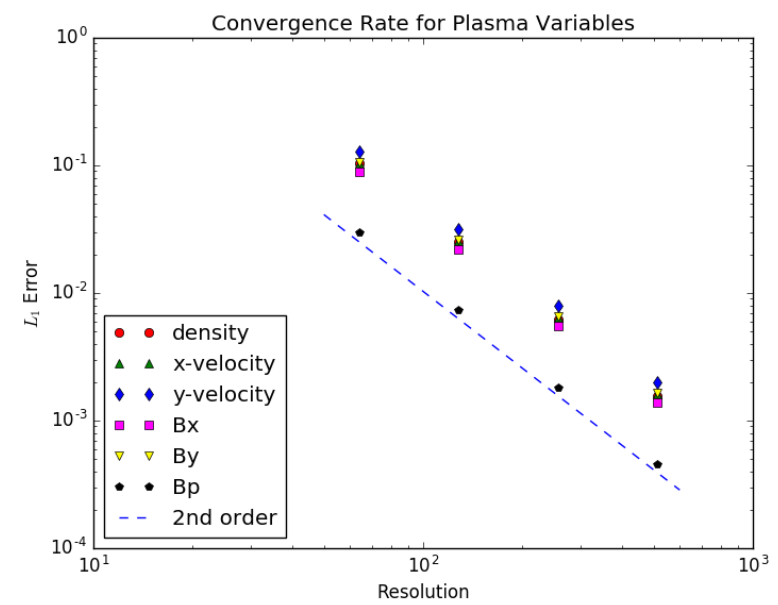

Figure 9: Convergence test of the 2D isentropic MHD vortex advection problem. The errors in $\rho, u, v, B_{x}, B_{y}$ and the magnetic pressure $B_{p}$ are calculated in $L_{1}$ sense against the initial conditions. The tested PCM solutions are solved on $N_{x} \times N_{y}$, where $N_{x}=N_{y}=32,64,128,256$ and 512. All runs reached to $t=10$ using the HLLC Riemann solver with $C_{\mathrm{cfl}}=0.8$.

convergence rate in 2D. We omit the details of the initial problem setup which can be found in [71, 3].

As expected, the results presented in Fig. 8 clearly confirm that, when $\mathrm{PCM}$ is extended to 2D using the simple dimension-by-dimension formulation, the overall numerical solution accuracy converges in second-order, regardless of its inherent fifth-order property in 1D.

\subsubsection{D Convergence Test of the Isentropic Vortex Advection - MHD case}

We also ran an MHD 2D isentropic vortex test which is an analogous MHD version of the previous hydro vortex test, in order to demonstrate PCM's convergence rate on the magnetic fields as well. The test was presented by Balsara 72] and we omit the details of the setup for brevity. As expected, the results presented in Fig. 9 clearly confirm that, PCM demonstrate second order accuracy in two dimensional magnetic field evolutions. 
(a) Sedov. We consider the Sedov blast test [73] to check PCM's ability to handle a spherical symmetry of the strong hydrodynamical shock explosion. The problem studies a self-similar evolution of a spherical shock wave propagation due to an initial point-source of a highly pressurized perturbation. The test has figurations were conjectured on polytropic gas. This claim was corrected by Schultz-Rinne [77] that one of them was impossible, and the numerical testings for such 15 configurations were studied in [78]. Later, Lax and Liu showed that there are total of 19 genuinely different configurations available, providing nu- 


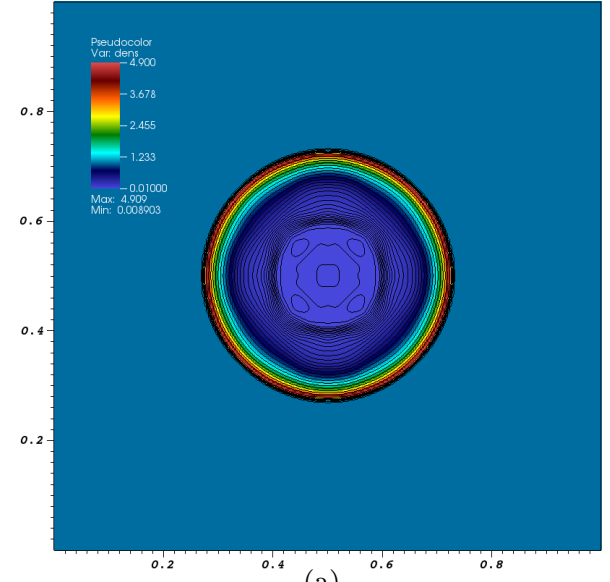

(a)

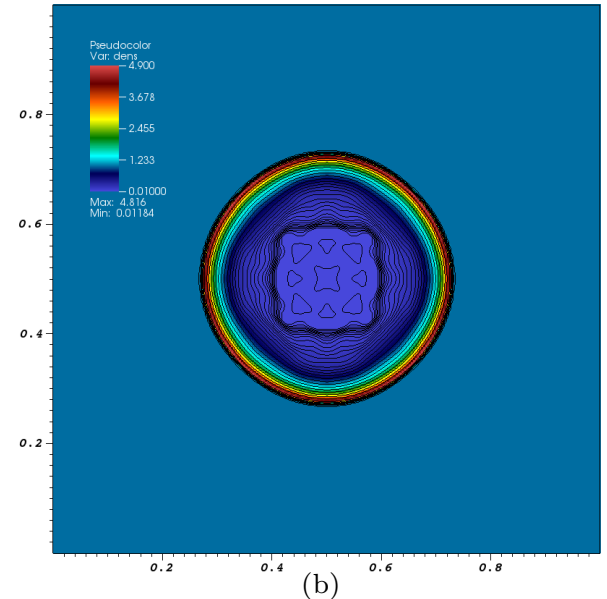

(b)

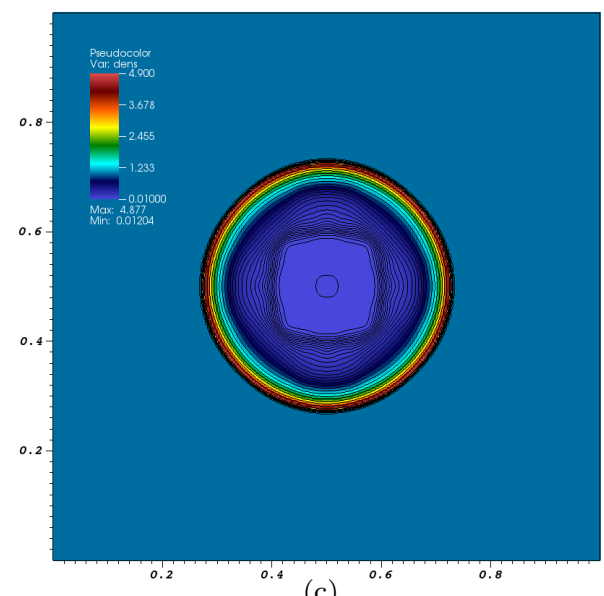

(c)

Figure 10: The Sedov explosion test. (a) PCM, (b) PPM, and (c) WENO-JS + charTr. All calculations are done on $256 \times 256$ cells with the HLLC Riemann solver and with $C_{\mathrm{cfl}}=0.8$. PPM is computed using the MC slope limiter. 30 equally spaced levels of density contour lines are shown in logarithmic scale.

merical solutions of all 19 cases too [79. See also [80. Until today, this family of 2D Riemann problems has been chosen by many people to demonstrate that their numerical algorithms can predict these 19 configurations successfully in pursuance of code verification purposes $[12,81,82,83$. We follow the setup as described in 82 in the following two verification tests, Configuration 3 and ${ }_{605}$ Configuration 5. In both cases the calculations show numerical solutions on 

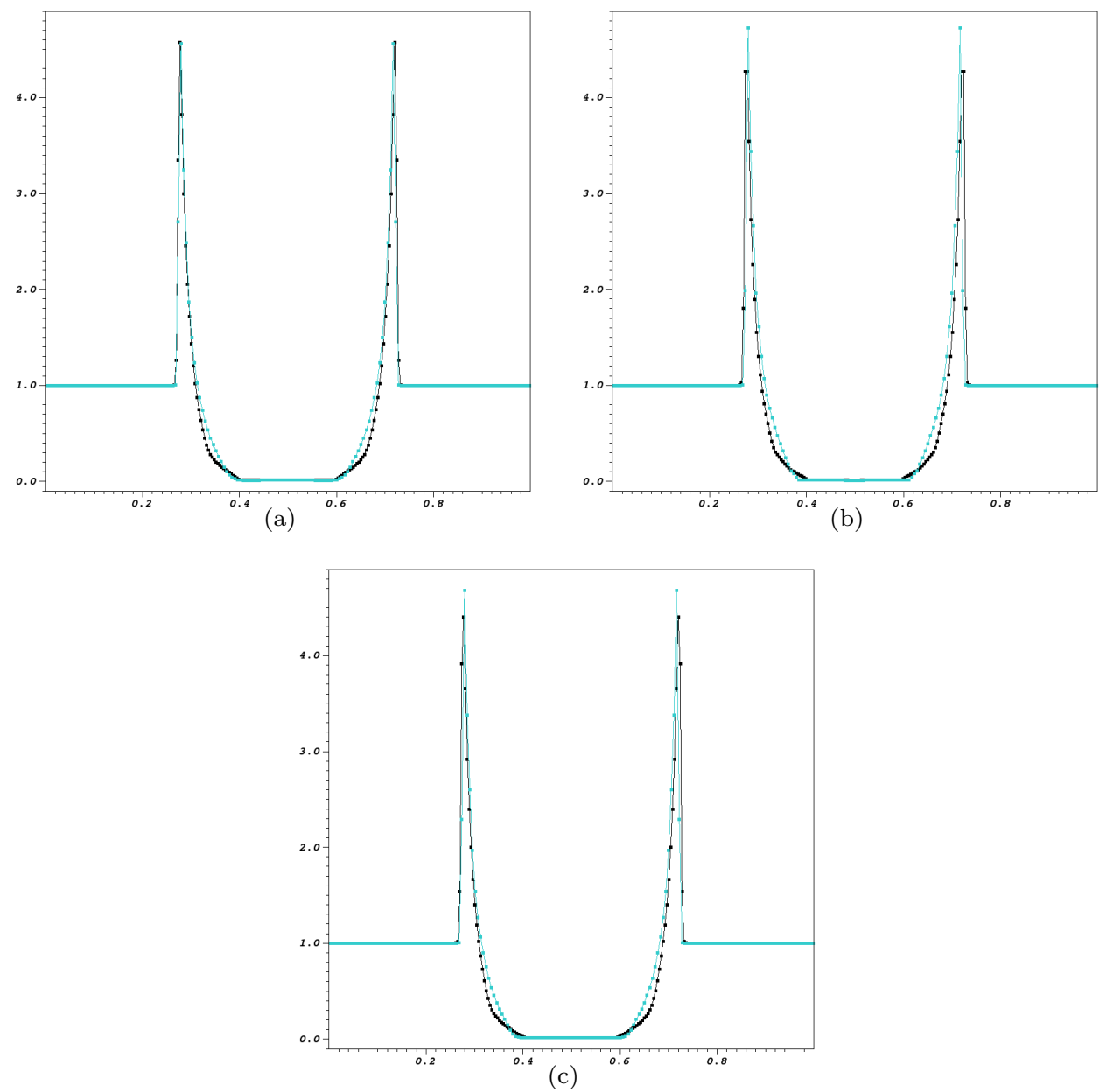

Figure 11: Two section cuts of $\rho$ from the Sedov test in Fig. 10 The two lines represent section cuts of densities along $y=0.5$ (black curves) and $y=x$ (cyan curves). (a) PCM, (b) PPM, and (c) WENO-JS + charTr.

$[0,1] \times[0,1]$ using outflow boundary conditions.

(b) - Configuration 3. Panels in Fig. 12 show numerical solutions of density at $t=0.8$ resolved on $400 \times 400$ using the HLLC Riemann solvers with $C_{\mathrm{cfl}}=0.8$. Also shown are the 40 contour lines of $\rho$. The range of $\rho$ is fixed as $0.1 \leq \rho \leq 1.8$ in both the pseudo-color figures and the contour lines. For the PCM method we employed both approaches of WENO-JS (see Eq. 433) and WENO-Z (see 


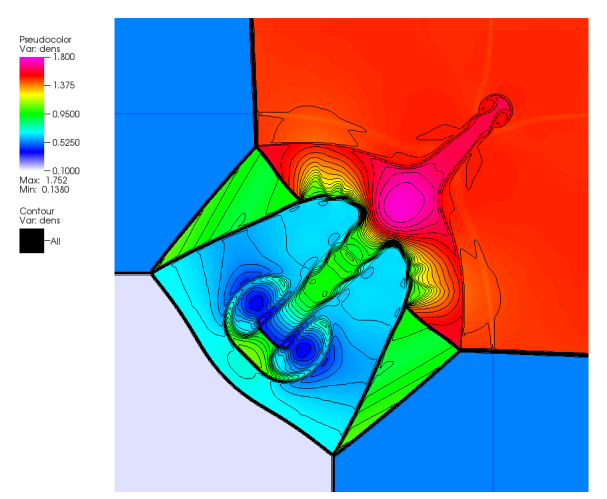

(a)

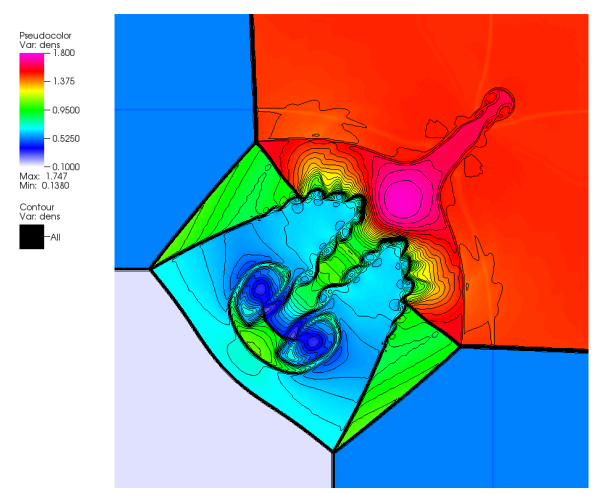

(c)

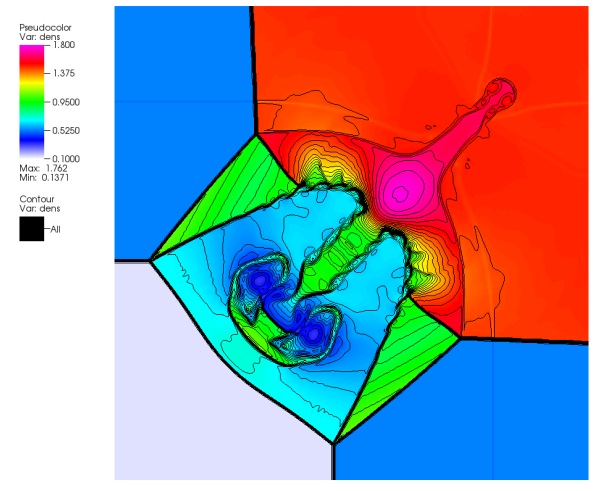

(e)

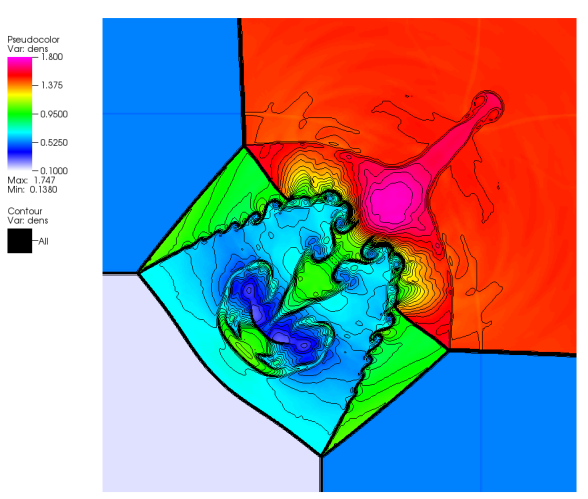

(b)

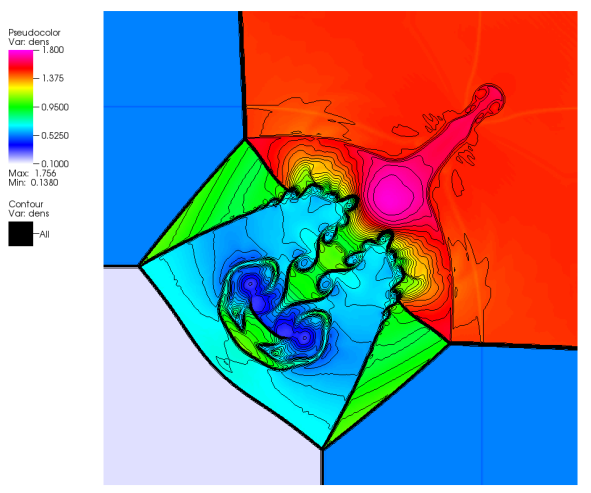

(d)

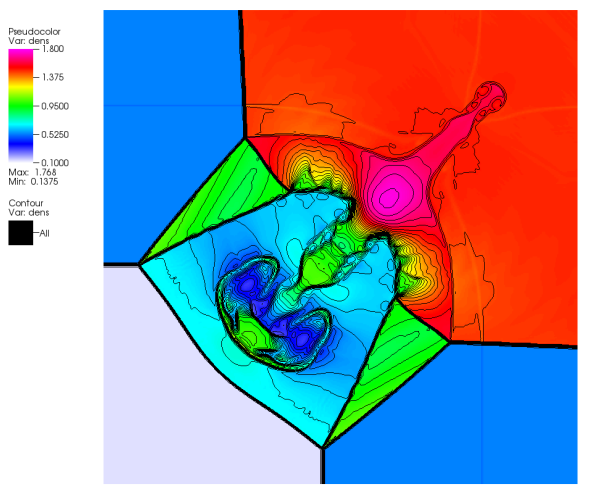

(f)

Figure 12: 2D Riemann Problem - Configuration 3. (a) PLM, (b) PPM, (c) WENO-JS + CharTr, (d) WENO-Z + CharTr, (e) PCM-JS, and (f) PCM-Z. Each panel shows the density values at $t=0.8$ between $[0.1,1.8]$ in linear scale, calculated using $400 \times 400$ grid cells. The total of 40 contour lines are over-plotted. The MC slope limiter is used in (a) and (b). 
Eq. (46)) for the calculations of the smoothness stencils. The figures can be directly compared with Fig. 6 in [82] where they used the same grid resolution for their hybrid compact-WENO scheme. First of all, including the two PCM solutions, we see that all calculations have produced their solutions successfully, in particular, without suffering unphysical oscillations near shocks and contact discontinuities. This test confirms that the PCM results are well comparable to the other solutions, except for some expected minor discrepancies.

One thing to notice is that the PPM solution in Fig. 12(b) has interestingly much more formations of Kelvin-Helmholtz instabilities, identified as vortical rollups along the slip lines (shown as the interface boundaries between the green triangular regions and the sky blue areas surrounding the mushroom-shaped jet). This feature is also often found in a test known as "Double Mach reflection" [57, where the similar pattern of rollups are detected along the slip line. As found in various studies [13, 30, 37] it is conventional to say that the amount of such vortical rollups at slip lines is one of the key factors to measure inherent numerical dissipations in a code. If we follow this approach, it then leads us to say that the PPM method is the least dissipative method among the six methods we tested. However, we find that this conclusion is somewhat arguable considering the nominal order of accuracy of PPM is lower than those of WENOJS + CharTr, WENO-Z + CharTr and PCM. We think that there is to be more accurate assessment regarding this type of conclusion. Readers can find a very similar numerical comparison between PPM and WENO-JS in [37.

(b) - Configuration 5. As a second 2D Riemann problem we consider Configura635 tion 5 to test PCM and compare its solution with three other solutions of PLM, PPM and WENO-JS + CharTr. The WENO-JS approaches in Eqs. 26) and (43) are adopted for PCM for the calculations of the smoothness stencils. The choice of grid resolution is $1024 \times 1024$ in this test in order to directly compare our results with the results reported in Fig. 4 and Fig. 5 of [12. As obtained in Fig. 13, the PCM solution satisfactorily compares well with the solutions of PPM and WENO-JS + CharTr, as well as with the high-resolution results 


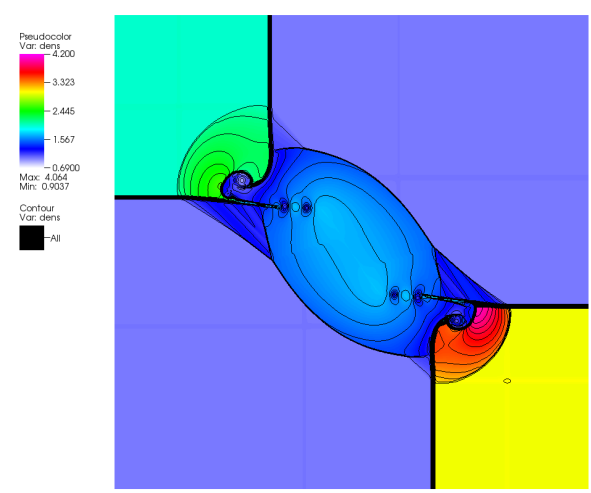

(a)

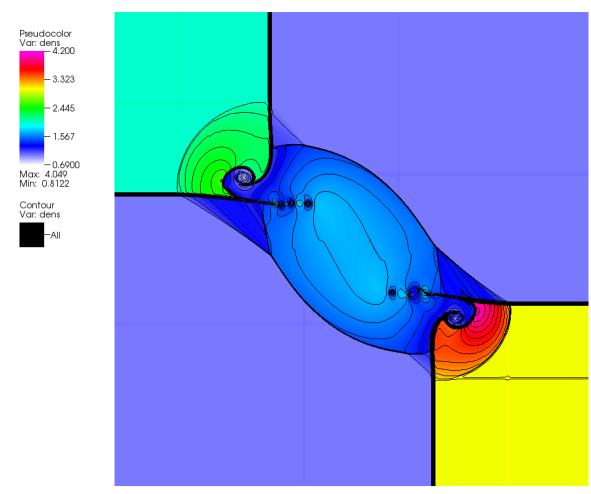

(c)

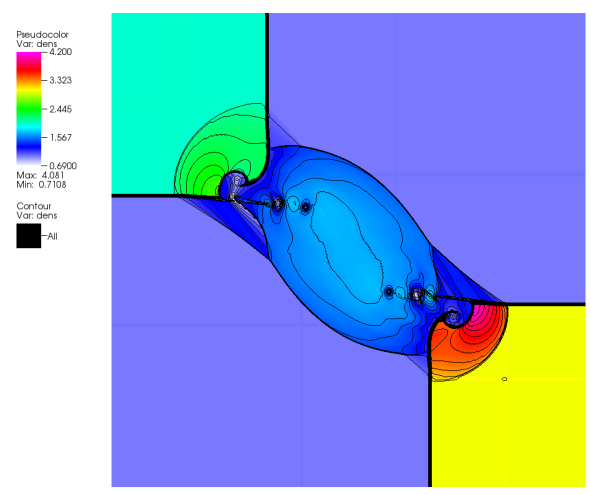

(b)

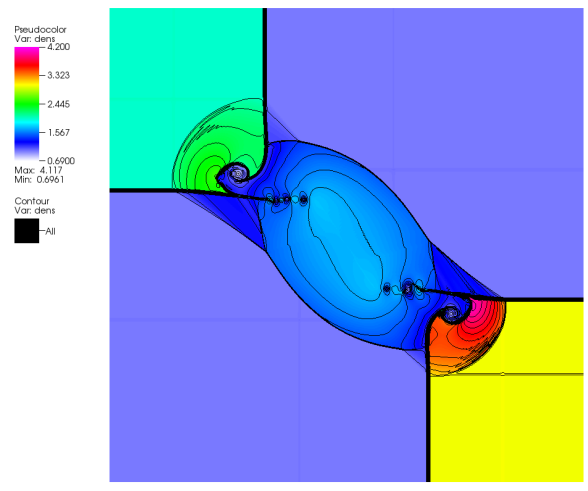

(d)

Figure 13: 2D Riemann Problem - Configuration 5. (a) PLM, (b) PPM, (c) WENO-JS + CharTr., and (d) PCM-JS. Each panel shows the density values at $t=0.23$ between $[0.69,4.2]$ in linear scale, resolved on $1024 \times 1024$ grid cells. The total of 40 contour lines are overplotted. The MC slope limiter is used in (a) and (b). All runs used the Roe Riemann solver with $C_{\text {cfl }}=0.8$. 
in [12. As also reported in [12, 13, when considering discontinuous flows in multiple space dimensions, the dimension-by-dimension approach works just as fine in terms of producing comparably accurate solutions. Likewise, the results in Fig. 13 show that we observe the same qualitative performances in all the methods we tested here, including PCM.

Although all the solutions are comparably admissible, there are few distinctive features in the PCM solution, displayed in Fig. 13 (d). We note that the minimum and maximum values of the computed density are respectively the smallest and the largest among the four results. This trend is consistent with the increasing order of accuracy in the four panels here. The same observation can be found also in Fig. 12 too. We consider this as an indication that the small scale features are better resolved in PCM with less amount of numerical diffusion. (c) MHD Rotor. Next, we consider the MHD rotor problem [84, 85]. As the problem has been discussed by various people we rather focus on discussing the solution of PCM here. We use the same setup conditions as described in [43]. Exhibited in Fig. 14(e) and Fig. 14(f) are respectively the density and the 30 contour lines of the Mach number on $400 \times 400$ cells, both at $t=0.15$. With minor discrepancies, we see that the PCM solution successfully demonstrates its ability to solve MHD flows in multiple space dimensions. To test PCM for multidimensional MHD flows, we integrated the PCM algorithm in the MHD scheme [43, 44] of the FLASH code [41, 42, 48]. Of noteworthy point is that the contour lines of the Mach number in Fig. 14(f) remain concentric in the central region without any distortion from the near-perfect symmetry. In all runs the HLLD Riemann solver [69] was used with a fixed value $C_{\mathrm{cfl}}=0.8$. The PPM run used the MC slope limiter for monotonicity.

\section{3. $3 D$ Tests}

Lastly, for 3D cases, we have selected three test problems in MHD in such 670 a way that we can fully quantify the performance of PCM both for assessing 

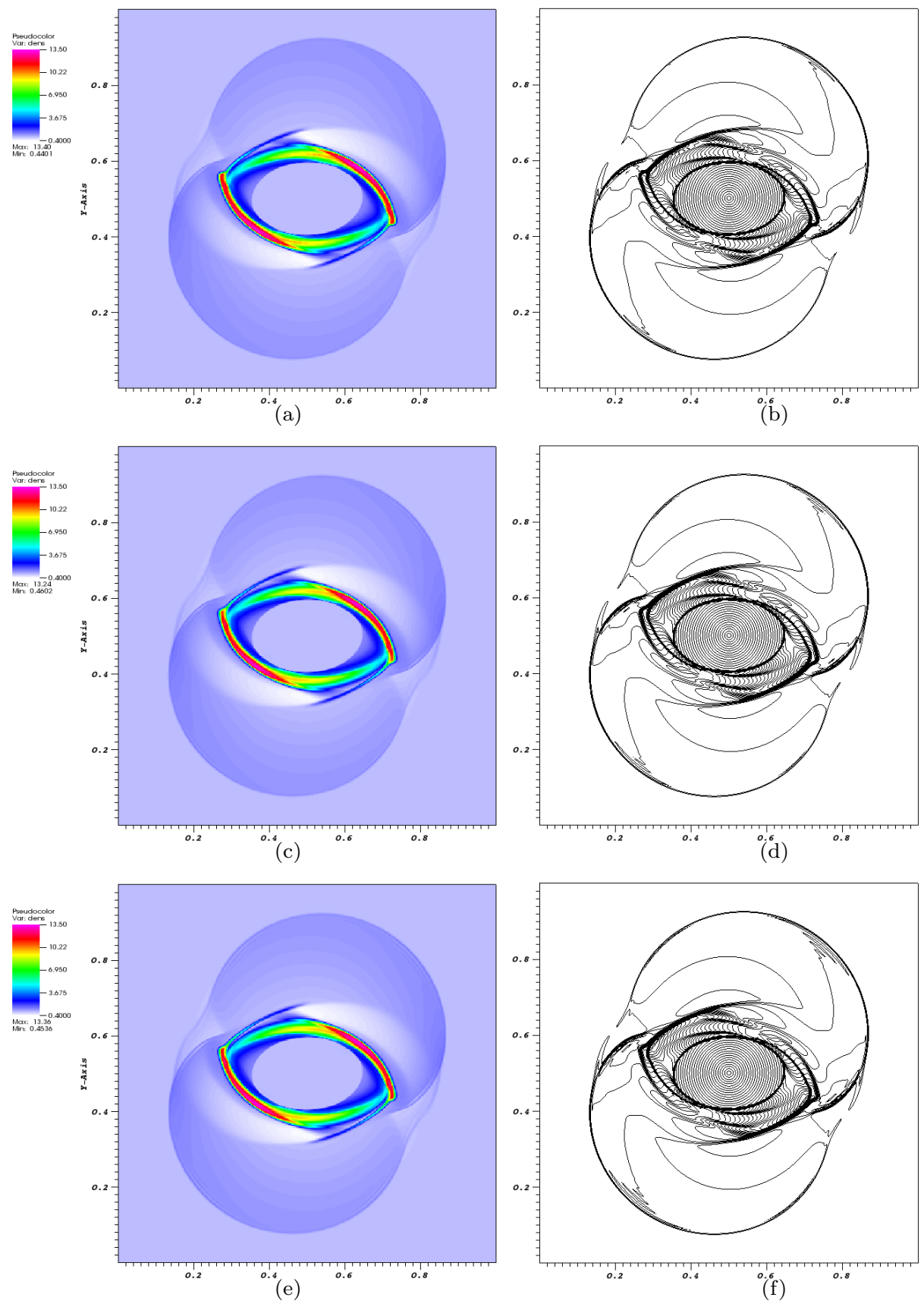

Figure 14: 2D MHD Rotor problem. The panels on the left column show the density at $t=0.15$, and the panels on the right show the 30 contour lines of the Mach number at the same time. (a) $\rho$ using PPM, (b) Mach number using PPM, (c) $\rho$ using WENO-JS + CharTr, (d) Mach number using WENO-JS + CharTr, (e) $\rho$ using PCM, and (f) Mach number using PCM. 
its convergence rate in $3 \mathrm{D}$ and for verifying its code capability in discontinuous flows.

\subsubsection{D Convergence Test}

Alfvén Wave Convergence Test-UG. We solve the circularly polarized Alfvén

675 Wave propagation problem [44, 50] as our first 3D test problem to quantify the PCM's order of accuracy in full 3D. The computational domain is resolved on $2 N_{x} \times N_{y} \times N_{z}$ grid cells, where we choose $N_{x}=N_{y}=N_{z}=8,16,32$ and 64 for the grid convergence study. As in [4] we ran the same two configurations of the wave mode that are the standing wave mode and the traveling wave mode until $t=1$. In both we choose the Roe Riemann solver with $C_{\mathrm{cfl}}=0.95$.

Respectively, Fig. 15(a) and Fig. 15(b) are the $L_{1}$ numerical errors on a logarithmic scale for the standing wave case and the traveling wave case. We observe that the rate of $\mathrm{PCM}$ convergence in $3 \mathrm{D}$ is second-order as expected, which agrees with the results reported in [44. One difference is noted in the standing wave case in Fig. 15(a) that PCM's $L_{1}$ error in each grid resolution is much lower than those obtained with PPM + HLLD + F-CTU with $C_{\mathrm{cfl}}=0.95$ in [44. However, the magnitudes of the PCM error in the traveling wave case in Fig. 15.(b) look pretty much similar to the equivalent run in [44.

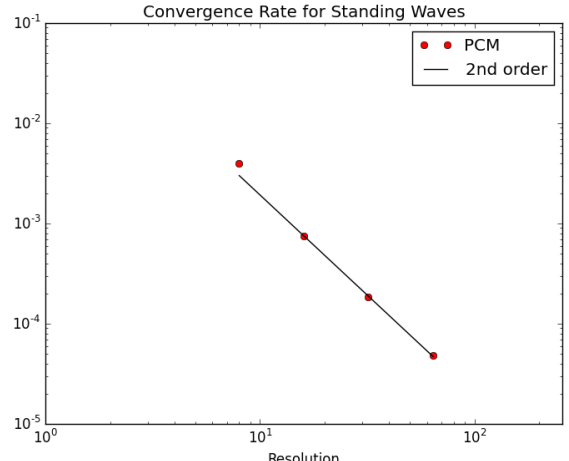

(a)

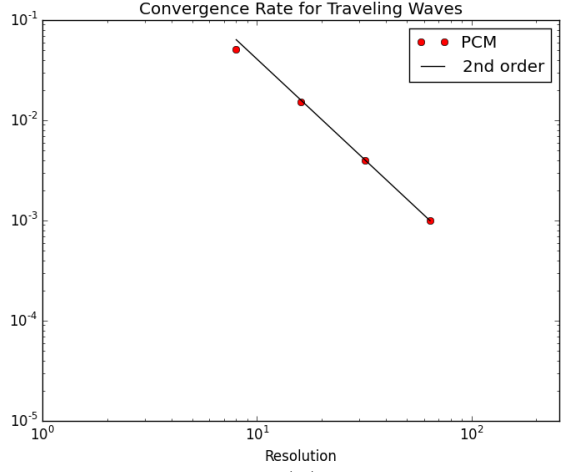

(b)

Figure 15: The circularly polarized Alfvén wave convergence rate for both the standing and traveling wave problems using PCM combined with the Roe Riemann solver. 


\subsubsection{D Discontinuous Tests}

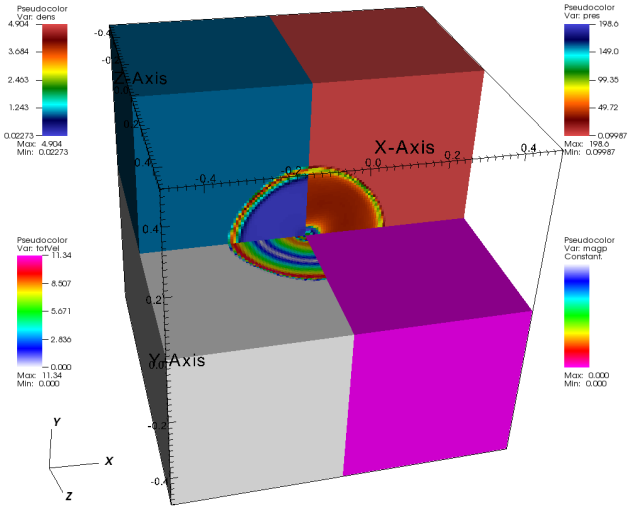

(a)

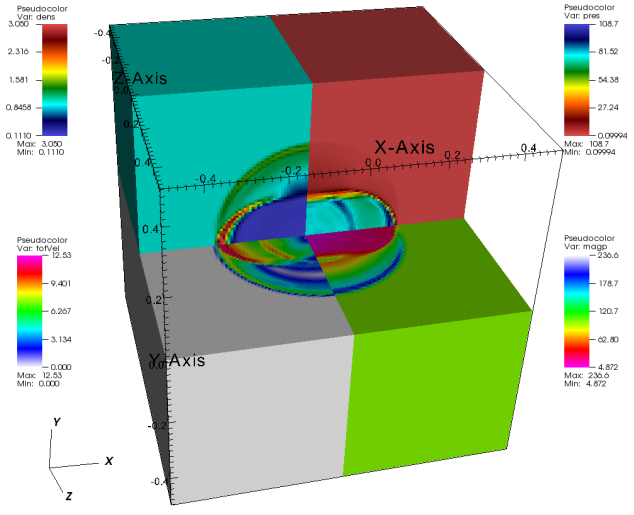

(b)

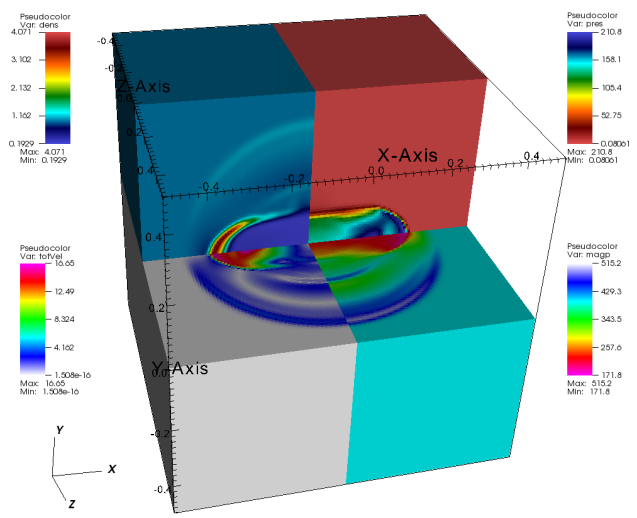

(c)

Figure 16: The 3D MHD blast problem. All results used the PCM scheme with the hybrid Riemann solver using $C_{\text {cfl }}=0.8$. (a) $B_{x}=0$, (b) $B_{x}=\frac{50}{\sqrt{4 \pi}}$, and (c) $B_{x}=\frac{100}{\sqrt{4 \pi}}$. In each panel, we show four different plasma quantities, gas pressure in the top right quadrant, density in top left, total velocity in bottom left, and magnetic pressure in bottom right. Each corresponding color bar is shown immediately next to the corresponding quantity.

(a) $3 D$ MHD Blast - UG. We consider the 3D variant of the MHD blast problem by adopting the setup conditions in [44] to demonstrate the three-dimensional propagation of strong MHD shocks using the PCM algorithm. The original 2D version of the spherical blast wave problem was studied in [86], and later various 
people adopted the similar setup conditions $440,43,44,84,87,88,89,90,91$, 92, 93 for their code verifications in strongly magnetized shock flows.

We display four different fluid variables in each panel in Fig. 16. From the top right quadrant to the bottom right quadrant in counter clockwise direction, we show the gas pressure $p$, the density $\rho$, the total velocity $U=\sqrt{u^{2}+v^{2}+w^{2}}$, and the magnetic pressure $B_{p}$, all plotted at $t=0.01$. The grid resolution $128 \times 128 \times 128$ as well as all other parameters are chosen as same as in 44 in order to provide a direct comparison.

We tested PCM in three different plasma conditions defined by the three different strengths of $B_{x}=0, \frac{50}{\sqrt{4 \pi}}$, and $\frac{100}{\sqrt{4 \pi}}$, as displayed in Fig. 16(a) $\sim$ Fig. 16 (c). Of particular interest to note is with the initial low plasma $\beta$ conditions 705 in the last two cases, $\beta=1 \times 10^{-3}$ and $2.513 \times 10^{-4}$, respectively. On the other hand, the first setup in Fig. 16.a) produces the non-magnetized plasma flow, hence it allows us to test the PCM algorithm in the pure hydrodynamical limit in 3D. As clearly seen, all results have produced confidently accurate solutions. We also note that PCM has produced larger values of extrema in each variable than those reported in [44], without exhibiting any unphysical oscillations. This test demonstrate that the PCM algorithm is well-suited for simulating low- $\beta$ flows in full 3D.

(b) Magnetic Field Loop Advection - UG. Since this problem was originally studied and reported in [94, the problem has become a popular benchmark case among various code developers to demonstrate their MHD algorithms' capabilities in advecting the initial field loop which is weakly magnetized with a very high plasma $\beta=2 \times 10^{6}$. The problem is known to be challenging [50, 94], however, many have demonstrated that their codes can successfully produced comparable results $[43,44,83,91,21,95,96]$. In addition to the two original setups [50, 94, where the initial field loops advect with the angle diagonal to the domain, Lee 44 recently reported that a small-angle advection is much more challenging. As an example, Lee adopted the advection angle $\theta \approx 0.573^{\circ}$ relative to the $x$-axis for the small-angle advection case in both $2 \mathrm{D}$ and $3 \mathrm{D}$. The 


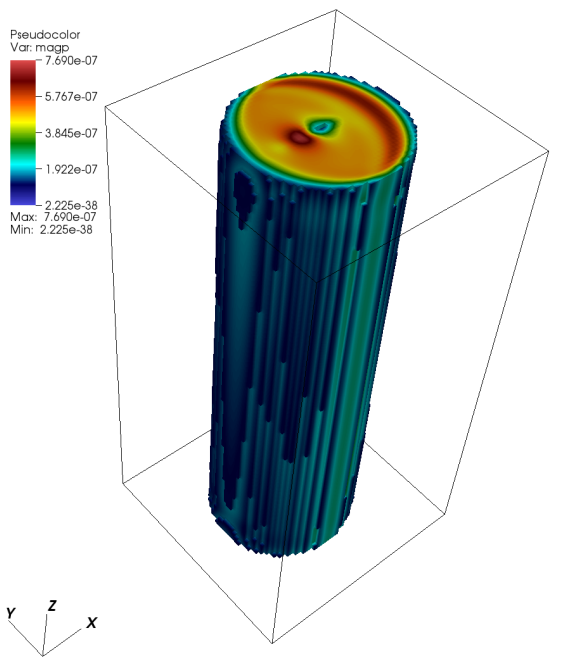

(a)

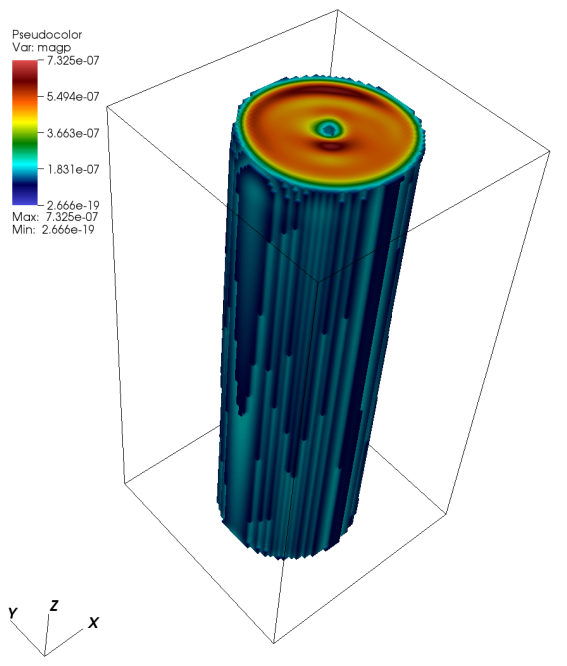

(b)

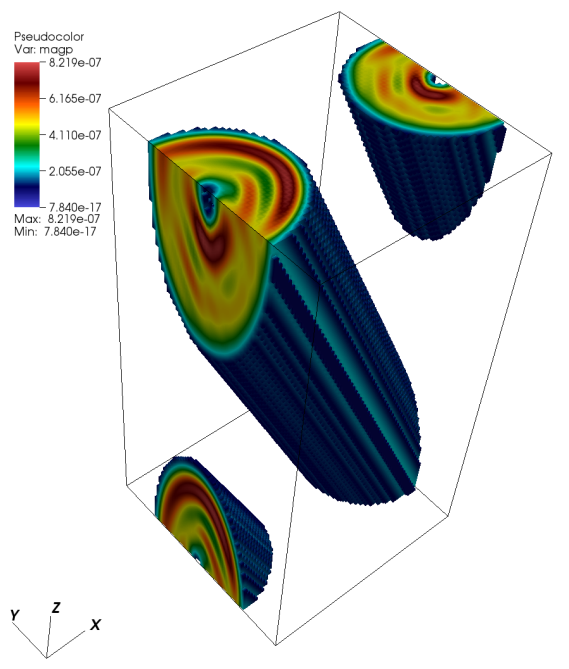

(c)

Figure 17: The 3D field loop advection using PCM. (a) the small angle advection with $\theta \approx$ $0.537^{\circ}$ at $t=2$, (b) the large angle advection at $t=2$ using $\mathbf{U}=(1,1,2)^{T}$, and (c) the standard Gardiner-Stone advection at $t=1$. All runs were calculated on $64 \times 64 \times 128$ cells using the upwind-MEC. 
study found that a proper amount of multidimensional numerical dissipation

plays a key role in maintaining the clean small-angle advection, and designed the algorithm called upwind-MEC. Here we repeat all three configurations (two large-angle advection cases and one small-angle advection case) by following the same setups in [44]. All the results in Fig. 17 were obtained using PCM and the Roe Riemann solver with $C_{\text {cfl }}=0.8$ on $64 \times 64 \times 128$ cells.

First, Fig. 17(a) shows the small-angle advection with $\theta \approx 0.573^{\circ}$ relative to the $x$-axis with the velocity fields given by $\mathbf{U}=(\cos \theta, \sin \theta, 2)^{T}$. Compared to this, in Fig. 17(b), we use $\mathbf{U}=(1,1,2)^{T}$ which yields the large-angle advection. In both cases the tilt angle $\omega$ (see [44 for details) is set to be same as $\theta$. As manifested, both runs cleanly preserve the initial geometry of the field loop, convincing us that the PCM algorithm is robust and accurate in this challenging problem. As a final test we also perform the standard field loop advection setup of Gardiner and Stone [50. The result is shown in Fig. 17(c). We see clearly that the PCM algorithm has produced well-behaving, accurate and confident solutions in this test. The results in Fig. 17 can be directly compared to the results reported in [4].

\section{Conclusions}

We summarize key features of the PCM algorithm studied in this paper.

- We have presented a new high-order finite volume scheme for the solutions of the compressible gas dynamics and ideal MHD equations in 1D. This baseline 1D algorithm uses piecewise cubic polynomials for spatial reconstruction by adopting the non-oscillatory approximations of the fifth order WENO schemes to determine the unique piecewise cubic polynomial on each cell. To provide the nominal fifth-order accuracy in space, we have developed a new non-oscillatory WENO-type reconstruction for $q_{C, i}^{\prime}$ approximation. The new approach makes use of the two parabolic polynomials, termed as PPM-Build, to achieve fourth-order accuracy in establishing $q_{C, i}^{\prime}$ approximation. 
- We have formulated a new fourth-order temporal updating scheme, all integrated in PCM by design, based on the simple predictor-corrector type characteristic tracing approach. The overall solution accuracy of the baseline 1D PCM scheme, combining both spatially and temporally, seemingly converges with fifth-order. We show the PCM scheme compares greatly with the spatially fifth-order WENO integrated with RK4, demonstrating even smaller $L_{1}$ errors in PCM.

- A comparison of the computational expenses of PCM, PPM and WENO$\mathrm{JS}+\mathrm{RK} 4$ in 1D reveals that PCM has a superior advantage over the fifth-order counterpart WENO-JS + RK4 by a factor of 1.71 .

- We have integrated the baseline 1D PCM algorithm for multidimensional cases by adopting the simple dimension-by-dimension approach. As anticipated, this approach yields at most second-order accurate solutions in multidimensional simulations of smooth flows. In the presence of flow discontinuities and shocks, however, the results obtained with the present simple multidimensional PCM extension shows a great level of confidence in predicting numerical solutions of hydrodynamics and MHD. An approach to extend the fifth-order property of the baseline 1D PCM to multiple spatial dimensions will be further investigated in our future work.

\section{Acknowledgements}

The software used in this work was in part developed by the DOE NNSAASC OASCR Flash Center at the University of Chicago. D. Lee also gratefully acknowledges the FLASH group for supporting the current work. The author also thank D. S. Balsara and an anonymous referee for very helpful suggestions and comments on the manuscript. 


\section{Appendix A. Reconstruction of the Riemann States $q_{L, i}$ and $q_{R, i}$ at fifth order}

We use the standard fifth order WENO reconstruction procedure to obtain the Riemann state values at cell interfaces. On each cell $I_{i}=\left[x_{i-1 / 2}, x_{i+1 / 2}\right]$, they can be explicitly written as

$$
q_{L ; R, i}=\sum_{\ell=1}^{3} \omega_{\ell}^{ \pm} p_{\ell}\left(x_{i \pm 1 / 2}\right),
$$

where

$$
\begin{aligned}
& p_{1}\left(x_{i-1 / 2}\right)=-\frac{1}{6} \bar{q}_{i-2}+\frac{5}{6} \bar{q}_{i-1}+\frac{1}{3} \bar{q}_{i}, \\
& p_{2}\left(x_{i-1 / 2}\right)=\frac{1}{3} \bar{q}_{i-1}+\frac{5}{6} \bar{q}_{i}-\frac{1}{6} \bar{q}_{i+1}, \\
& p_{3}\left(x_{i-1 / 2}\right)=\frac{11}{6} \bar{q}_{i}-\frac{7}{6} \bar{q}_{i+1}+\frac{1}{3} \bar{q}_{i+2},
\end{aligned}
$$

and

$$
\begin{aligned}
& p_{1}\left(x_{i+1 / 2}\right)=\frac{1}{3} \bar{q}_{i-2}-\frac{7}{6} \bar{q}_{i-1}+\frac{11}{6} \bar{q}_{i}, \\
& p_{2}\left(x_{i+1 / 2}\right)=-\frac{1}{6} \bar{q}_{i-1}+\frac{5}{6} \bar{q}_{i}+\frac{1}{3} \bar{q}_{i+1}, \\
& p_{3}\left(x_{i+1 / 2}\right)=\frac{1}{3} \bar{q}_{i}+\frac{5}{6} \bar{q}_{i+1}-\frac{1}{6} \bar{q}_{i+2} .
\end{aligned}
$$

The nonlinear weights $\omega_{\ell}^{ \pm}$are constructed based on one of the WENO-JS, WENO-M, or WENO-Z schemes in Eqs. 26) $\sim 29$. The smoothness indicators in Eqs. $262 \sim 29$ are given by following the standard fifth order WENO reconstruction [11:

$$
\begin{aligned}
& \beta_{1}=\frac{13}{12}\left(\bar{q}_{i-2}-2 \bar{q}_{i-1}+\bar{q}_{i}\right)^{2}+\frac{1}{4}\left(\bar{q}_{i-2}-4 \bar{q}_{i-1}+3 \bar{q}_{i}\right)^{2}, \\
& \beta_{2}=\frac{13}{12}\left(\bar{q}_{i-1}-2 \bar{q}_{i}+\bar{q}_{i+1}\right)^{2}+\frac{1}{4}\left(\bar{q}_{i-1}-\bar{q}_{i+1}\right)^{2}, \\
& \beta_{3}=\frac{13}{12}\left(\bar{q}_{i}-2 \bar{q}_{i+1}+\bar{q}_{i+2}\right)^{2}+\frac{1}{4}\left(3 \bar{q}_{i}-4 \bar{q}_{i+1}+\bar{q}_{i+2}\right)^{2} .
\end{aligned}
$$

\section{Appendix B. Reconstruction of the derivative $q_{C, i}^{\prime}$ at fourth order}

We provide explicit forms to determine $q_{C, i}^{\prime}$ at fourth order on the five-point stencil $S=\left\{I_{i-2}, \ldots, I_{i+2}\right\}$. The stencil $S$ is subdivided into two four-point 
sub-stencil $S_{1}=\left\{I_{i-2}, \ldots, I_{i+1}\right\}$ and $S_{2}=\left\{I_{i-1}, \ldots, I_{i+2}\right\}$ so that $S=\cup_{\ell=1}^{2} S_{\ell}$. This strategy is based on the $r=4$ WENO description of Balsara and Shu [3], and is slightly modified to obtain the slope $q_{C, i}^{\prime}$ in our case. Here we provide detailed expressions and derivations in Step 1 and Step 2 in Section 2.3.

Step 1. The coefficients of $\phi_{ \pm}$in Eq. (36) are given as

$$
\begin{aligned}
a_{0}^{ \pm} & =\frac{1}{12}\left(-\bar{q}_{i-2+s}+7 \bar{q}_{i-1+s}+7 \bar{q}_{i+s}-\bar{q}_{i+1+s}\right), \\
a_{1}^{ \pm} & =\frac{1}{12 \Delta x}\left(\bar{q}_{i-2+s}-15 \bar{q}_{i-1+s}+15 \bar{q}_{i+s}-\bar{q}_{i+1+s}\right), \\
a_{2}^{ \pm} & =\frac{1}{4 \Delta x^{2}}\left(\bar{q}_{i-2+s}-\bar{q}_{i-1+s}-\bar{q}_{i+s}+\bar{q}_{i+1+s}\right), \\
a_{3}^{ \pm} & =\frac{1}{6 \Delta x^{3}}\left(-\bar{q}_{i-2+s}+3 \bar{q}_{i-1+s}-3 \bar{q}_{i+s}+\bar{q}_{i+1+s}\right),
\end{aligned}
$$

where $s=1$ for $a_{k}^{+}$, while $s=0$ for $a_{k}^{-}$.

Step 2. To determine the two linear weights $\gamma_{ \pm}$in Eq. (37), we first define the fourth degree polynomial $\phi(x)$ over the five-point stencil $S$,

$$
\phi(x)=\sum_{k=0}^{4} b_{k}\left(x-x_{i}\right)^{k} .
$$

Now $\gamma_{ \pm}$are determined by solving

$$
\gamma_{-} \phi_{-}^{\prime}\left(x_{i}\right)+\gamma_{+} \phi_{+}^{\prime}\left(x_{i}\right)=\phi^{\prime}\left(x_{i}\right) .
$$

This is equivalent to solving

$$
\gamma_{-}\left(a_{1}^{-}+a_{2}^{-} \Delta x+3 a_{3}^{-} \frac{\Delta x^{2}}{4}\right)+\gamma_{+}\left(a_{1}^{+}-a_{2}^{+} \Delta x+3 a_{3}^{+} \frac{\Delta x^{2}}{4}\right)=b_{1}
$$

where $b_{1}$ is given explicitly as

$$
b_{1}=\frac{5}{48} \bar{q}_{i-2}-\frac{17}{24} \bar{q}_{i-1}+\frac{17}{24} \bar{q}_{i+1}-\frac{5}{48} \bar{q}_{i+2} .
$$

This results in $\gamma_{-}=\gamma_{+}=\frac{1}{2}$. 


\section{References}

805 [1] P. Colella, P. R. Woodward, The piecewise parabolic method (PPM) for gas-dynamical simulations, Journal of computational physics 54 (1) (1984) $174-201$.

[2] G.-S. Jiang, C.-W. Shu, Efficient implementation of weighted ENO schemes, Journal of Computational Physics 126 (1) (1996) 202-228.

[3] D. S. Balsara, C.-W. Shu, Monotonicity preserving weighted essentially non-oscillatory schemes with increasingly high order of accuracy, Journal of Computational Physics 160 (2) (2000) 405-452.

[4] J. J. Dongarra, H. W. Meuer, H. D. Simon, E. Strohmaier, Recent trends in high performance computing, The Birth of Numerical Analysis (2010) 93.

[5] J. Dongarra, On the Future of High Performance Computing: How to Think for Peta and Exascale Computing, Hong Kong University of Science and Technology, 2012.

[6] A. Subcommittee, Top ten exascale research challenges, US Department Of Energy Report, 2014.

[7] D. E. Keyes, L. C. McInnes, C. Woodward, W. Gropp, E. Myra, M. Pernice, J. Bell, J. Brown, A. Clo, J. Connors, et al., Multiphysics simulations challenges and opportunities, International Journal of High Performance Computing Applications 27 (1) (2013) 4-83.

[8] R. J. LeVeque, Finite volume methods for hyperbolic problems, Vol. 31, Cambridge university press, 2002.

[9] R. J. LeVeque, Finite difference methods for ordinary and partial differential equations: steady-state and time-dependent problems, Vol. 98, Siam, 2007. 
[10] E. Toro, Riemann Solvers and Numerical Methods for Fluid Dynamics: A Practical Introduction, Springer, 2009.

URL http://books.google.com/books?id=SqEjX0um8o0C

[11] C.-W. Shu, High order weighted essentially nonoscillatory schemes for convection dominated problems, SIAM review 51 (1) (2009) 82-126.

835 [12] P. Buchmüller, C. Helzel, Improved accuracy of high-order WENO finite volume methods on Cartesian grids, Journal of Scientific Computing 61 (2) (2014) 343-368.

[13] R. Zhang, M. Zhang, C.-W. Shu, On the order of accuracy and numerical performance of two classes of finite volume WENO schemes, Communications in Computational Physics 9 (03) (2011) 807-827.

[14] P. McCorquodale, P. Colella, A high-order finite-volume method for conservation laws on locally refined grids, Communications in Applied Mathematics and Computational Science 6 (1) (2011) 1-25.

[15] V. A. Titarev, E. F. Toro, Ader: Arbitrary high order godunov approach, Journal of Scientific Computing 17 (1) (2002) 609-618.

[16] E. F. Toro, V. A. Titarev, Derivative riemann solvers for systems of conservation laws and ader methods, Journal of Computational Physics 212 (1) (2006) 150-165.

[17] V. Titarev, E. Toro, ADER schemes for three-dimensional non-linear hyperbolic systems, Journal of Computational Physics 204 (2) (2005) 715 - 736. doi:10.1016/j.jcp.2004.10.028. URL http://www.sciencedirect.com/science/article/pii/ S0021999104004358

[18] M. Dumbser, D. S. Balsara, E. F. Toro, C.-D. Munz, A unified framework 855 for the construction of one-step finite volume and discontinuous Galerkin schemes on unstructured meshes, Journal of Computational Physics 227 (2008) 8209-8253. doi:10.1016/j.jcp.2008.05.025. 
[19] D. S. Balsara, T. Rumpf, M. Dumbser, C.-D. Munz, Efficient, high accuracy ader-weno schemes for hydrodynamics and divergence-free magnetohydrodynamics, Journal of Computational Physics 228 (7) (2009) 2480-2516.

[20] M. Dumbser, O. Zanotti, A. Hidalgo, D. S. Balsara, Ader-weno finite volume schemes with space-time adaptive mesh refinement, Journal of Computational Physics 248 (2013) 257-286.

[21] D. S. Balsara, C. Meyer, M. Dumbser, H. Du, Z. Xu, Efficient implementation of ader schemes for euler and magnetohydrodynamical flows on structured meshes-speed comparisons with runge-kutta methods, Journal of Computational Physics 235 (2013) 934-969.

[22] J. Qian, J. Li, S. Wang, The generalized riemann problems for compressible fluid flows: Towards high order, Journal of Computational Physics 259 (2014) 358-389.

[23] M. Dumbser, M. Käser, V. A. Titarev, E. F. Toro, Quadrature-free nonoscillatory finite volume schemes on unstructured meshes for nonlinear hyperbolic systems, Journal of Computational Physics 226 (1) (2007) 204-243.

[24] C.-W. Shu, Total-variation-diminishing time discretizations, SIAM J. Sci. and Stat. Comput. 9 (6) (1988) 1073-1084.

[25] S. K. Godunov, A difference method for numerical calculation of discontinuous solutions of the equations of hydrodynamics, Matematicheskii Sbornik 47(89) (3) (1959) 271-306.

[26] J. Shi, C. Hu, C.-W. Shu, A technique of treating negative weights in WENO schemes, Journal of Computational Physics 175 (1) (2002) 108127.

[27] R. Borges, M. Carmona, B. Costa, W. S. Don, An improved weighted essentially non-oscillatory scheme for hyperbolic conservation laws, Journal of Computational Physics 227 (6) (2008) 3191-3211. 
[33] M. Brio, C. C. Wu, An upwind differencing scheme for the equations of ideal magnetohydrodynamics, Journal of computational physics 75 (2) (1988) $400-422$.

[34] A. K. Henrick, T. D. Aslam, J. M. Powers, Mapped weighted essentially non-oscillatory schemes: achieving optimal order near critical points, Journal of Computational Physics 207 (2) (2005) 542-567.

[35] D. S. Balsara, S. Garain, C.-W. Shu, An efficient class of weno schemes with adaptive order, Journal of Computational Physics 326 (2016) 780-804.

[36] P. Colella, A direct Eulerian MUSCL scheme for gas dynamics, SIAM Jour- 
[37] A. Mignone, G. Bodo, S. Massaglia, T. Matsakos, O. Tesileanu, C. Zanni, A. Ferrari, PLUTO: a numerical code for computational astrophysics, The Astrophysical Journal Supplement Series 170 (1) (2007) 228.

[38] A. Mignone, C. Zanni, P. Tzeferacos, B. van Straalen, P. Colella, G. Bodo, The PLUTO code for adaptive mesh computations in astrophysical fluid dynamics, The Astrophysical Journal Supplement Series 198 (1) (2011) 7.

[39] A. Mignone, P. Tzeferacos, A second-order unsplit Godunov scheme for cellcentered MHD: The CTU-GLM scheme, Journal of Computational Physics 229 (6) (2010) 2117-2138.

[40] J. M. Stone, T. A. Gardiner, P. Teuben, J. F. Hawley, J. B. Simon, Athena: a new code for astrophysical MHD, The Astrophysical Journal Supplement Series 178 (1) (2008) 137.

[41] B. Fryxell, K. Olson, P. Ricker, F. Timmes, M. Zingale, D. Lamb, P. MacNeice, R. Rosner, J. Truran, H. Tufo, FLASH: An adaptive mesh hydrodynamics code for modeling astrophysical thermonuclear flashes, The Astrophysical Journal Supplement Series 131 (1) (2000) 273.

[42] A. Dubey, K. Antypas, M. K. Ganapathy, L. B. Reid, K. Riley, D. Sheeler, A. Siegel, K. Weide, Extensible component-based architecture for FLASH, a massively parallel, multiphysics simulation code, Parallel Computing 35 (10) (2009) 512-522.

[43] D. Lee, A. E. Deane, An unsplit staggered mesh scheme for multidimensional magnetohydrodynamics, Journal of Computational Physics 228 (4) (2009) 952-975.

[44] D. Lee, A solution accurate, efficient and stable unsplit staggered mesh scheme for three dimensional magnetohydrodynamics, Journal of Computational Physics 243 (2013) 269-292. 
[45] G. L. Bryan, M. L. Norman, J. M. Stone, R. Cen, J. P. Ostriker, A piecewise parabolic method for cosmological hydrodynamics, Computer Physics Communications 89 (1) (1995) 149-168.

[46] G. L. Bryan, M. L. Norman, B. W. O'Shea, T. Abel, J. H. Wise, M. J. Turk, D. R. Reynolds, D. C. Collins, P. Wang, S. W. Skillman, et al., Enzo: An adaptive mesh refinement code for astrophysics, The Astrophysical Journal Supplement Series 211 (2) (2014) 19.

[47] R. Teyssier, Cosmological hydrodynamics with adaptive mesh refinement-a new high resolution code called RAMSES, Astronomy \&amp; Astrophysics 385 (1) (2002) 337-364.

[48] D. Lee, P. Tzeferacos, S. Couch, J. Bachan, D. C., A. Dubey, M. Fatenejad, N. Flocke, C. Graziani, D. Lamb, K. Weide, FLASH: A multi-physics code for adaptive mesh computational fluid dynamics in astrophysics, In preparation.

[49] P. Colella, Multidimensional upwind methods for hyperbolic conservation laws, Journal of Computational Physics 87 (1) (1990) 171-200.

[50] T. A. Gardiner, J. M. Stone, An unsplit Godunov method for ideal MHD via constrained transport in three dimensions, Journal of Computational Physics 227 (8) (2008) 4123-4141.

[51] J. Saltzman, An unsplit 3D upwind method for hyperbolic conservation laws, Journal of Computational Physics 115 (1) (1994) 153-168.

[52] E. F. Toro, M. Spruce, W. Speares, Restoration of the contact surface in the HLL-Riemann solver, Shock waves 4 (1) (1994) 25-34.

[53] G. A. Sod, A survey of several finite difference methods for systems of nonlinear hyperbolic conservation laws, Journal of computational physics 27 (1) (1978) 1-31. 
[54] P. L. Roe, Approximate Riemann solvers, parameter vectors, and difference schemes, Journal of computational physics 43 (2) (1981) 357-372.

[55] C.-W. Shu, S. Osher, Efficient implementation of essentially non-oscillatory shock-capturing schemes, II, Journal of Computational Physics 83 (1) (1989) 32-78.

[56] B. Einfeldt, C.-D. Munz, P. L. Roe, B. Sjögreen, On Godunov-type methods near low densities, Journal of computational physics 92 (2) (1991) 273-295.

[57] P. Woodward, P. Colella, The numerical simulation of two-dimensional fluid flow with strong shocks, Journal of computational physics 54 (1) (1984) $115-173$.

[58] M. Torrilhon, Uniqueness conditions for Riemann problems of ideal magnetohydrodynamics, Journal of plasma physics 69 (03) (2003) 253-276.

[59] M. Torrilhon, Non-uniform convergence of finite volume schemes for Riemann problems of ideal magnetohydrodynamics, Journal of Computational Physics 192 (1) (2003) 73-94.

[60] D. Lee, An upwind slope limiter for PPM that preserves monotonicity in magnetohydrodynamics, in: 5th International Conference of Numerical Modeling of Space Plasma Flows (ASTRONUM 2010), Vol. 444, 2011, p. 236.

[61] S. Karni, S. Čanić, Computations of slowly moving shocks, Journal of Computational Physics 136 (1) (1997) 132-139.

[62] Y. Stiriba, R. Donat, A numerical study of postshock oscillations in slowly moving shock waves, Computers \&amp; Mathematics with Applications 46 (5) (2003) 719-739.

[63] M. Arora, P. L. Roe, On postshock oscillations due to shock capturing schemes in unsteady flows, Journal of Computational Physics 130 (1) (1997) $25-40$. 
[64] S. Jin, J.-G. Liu, The effects of numerical viscosities: I. slowly moving shocks, Journal of Computational Physics 126 (2) (1996) 373-389.

[65] T. W. Roberts, The behavior of flux difference splitting schemes near slowly moving shock waves, Journal of Computational Physics 90 (1) (1990) 141160.

[66] E. Johnsen, S. Lele, Numerical errors generated in simulations of slowly moving shocks, Center for Turbulence Research, Annual Research Briefs (2008) 1-12.

[67] A. Harten, P. D. Lax, B. Van Leer, On upstream differencing and Godunovtype schemes for hyperbolic conservation laws, in: Upwind and HighResolution Schemes, Springer, 1997, pp. 53-79.

[68] S. Li, An HLLC Riemann solver for magneto-hydrodynamics, Journal of computational physics 203 (1) (2005) 344-357.

[69] T. Miyoshi, K. Kusano, A multi-state HLL approximate Riemann solver for ideal magnetohydrodynamics, Journal of Computational Physics 208 (1) (2005) 315-344.

[70] D. Ryu, T. Jones, Numerical magnetohydrodynamics in astrophysics: algorithm and tests for one-dimensional flow, arXiv preprint astro-ph/9404074.

[71] H. C. Yee, M. Vinokur, M. J. Djomehri, Entropy splitting and numerical dissipation, Journal of Computational Physics 162 (1) (2000) 33-81.

[72] D. S. Balsara, Second-order-accurate schemes for magnetohydrodynamics with divergence-free reconstruction, The Astrophysical Journal Supplement Series 151 (1) (2004) 149.

URL http://stacks .iop.org/0067-0049/151/i=1/a=149

[73] L. I. Sedov, Similarity and dimensional methods in mechanics, CRC press, 1993. 
[74] D. S. Balsara, A two-dimensional hllc riemann solver for conservation laws: Application to euler and magnetohydrodynamic flows, Journal of Computational Physics 231 (22) (2012) 7476-7503.

[75] D. S. Balsara, M. Dumbser, R. Abgrall, Multidimensional hllc Riemann solver for unstructured meshes-with application to Euler and MHD flows, Journal of Computational Physics 261 (2014) 172-208.

[76] T. Zhang, Y. X. Zheng, Conjecture on the structure of solutions of the Riemann problem for two-dimensional gas dynamics systems, SIAM Journal on Mathematical Analysis 21 (3) (1990) 593-630.

1025 [77] C. W. Schulz-Rinne, Classification of the Riemann problem for twodimensional gas dynamics, SIAM journal on mathematical analysis 24 (1) (1993) 76-88.

[78] C. W. Schulz-Rinne, J. P. Collins, H. M. Glaz, Numerical solution of the Riemann problem for two-dimensional gas dynamics, SIAM Journal on Scientific Computing 14 (6) (1993) 1394-1414.

[79] P. D. Lax, X.-D. Liu, Solution of two-dimensional Riemann problems of gas dynamics by positive schemes, SIAM Journal on Scientific Computing 19 (2) (1998) 319-340.

[80] T. Chang, G.-Q. Chen, S. Yang, On the 2-D Riemann problem for the compressible euler equations. I. Interaction of shocks and rarefaction waves, Discrete and Continuous Dynamical Systems 1 (1995) 555-584.

[81] A. Kurganov, E. Tadmor, Solution of two-dimensional Riemann problems for gas dynamics without Riemann problem solvers, Numerical Methods for Partial Differential Equations 18 (5) (2002) 584-608.

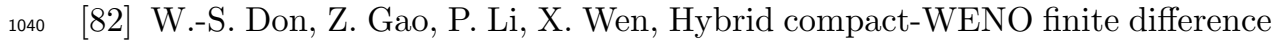
scheme with conjugate fourier shock detection algorithm for hyperbolic conservation laws, SIAM Journal on Scientific Computing 38 (2) (2016) A691-A711. 
[83] D. S. Balsara, Multidimensional HLLE Riemann solver: Application to Euler and magnetohydrodynamic flows, Journal of Computational Physics 229 (6) (2010) 1970-1993.

[84] D. Balsara, D. Spicer, A staggered mesh algorithm using high order Godunov fluxes to ensure solenoidal magnetic fields in magnetohydrodynamic simulations, Journal of Computational Physics 149 (2) (1999) 270-292.

[85] G. Tóth, The $\nabla \cdot B=0$ Constraint in Shock-Capturing Magnetohydrodynamics Codes, Journal of Computational Physics 161 (2) (2000) 605-652.

[86] D. Koessl, E. Mueller, W. Hillebrandt, Numerical simulations of axially symmetric magnetized jets. I-The influence of equipartition magnetic fields. II-Apparent field structure and theoretical radio maps. III-Collimation of underexpanded jets by magnetic fields, Astronomy and Astrophysics 229 (1990) 378-415.

[87] A. L. Zachary, A. Malagoli, P. Colella, A higher-order Godunov method for multidimensional ideal magnetohydrodynamics, SIAM Journal on Scientific Computing 15 (2) (1994) 263-284.

[88] D. S. Balsara, M. Dumbser, Divergence-free MHD on unstructured meshes using high order finite volume schemes based on multidimensional Riemann solvers, Journal of Computational Physics 299 (2015) 687-715.

[89] U. Ziegler, A semi-discrete central scheme for magnetohydrodynamics on orthogonal-curvilinear grids, Journal of Computational Physics 230 (4) (2011) 1035-1063.

[90] A. Mignone, P. Tzeferacos, G. Bodo, High-order conservative finite difference GLM-MHD schemes for cell-centered MHD, Journal of Computational Physics 229 (17) (2010) 5896-5920.

[91] S. Li, High order central scheme on overlapping cells for magnetohydrodynamic flows with and without constrained transport method, Journal of Computational Physics 227 (15) (2008) 7368-7393. 
[92] S. Kawai, Divergence-free-preserving high-order schemes for magnetohydrodynamics: An artificial magnetic resistivity method, Journal of Computational Physics 251 (2013) 292-318.

1075 [93] P. Londrillo, L. Del Zanna, High-order upwind schemes for multidimensional magnetohydrodynamics, The Astrophysical Journal 530 (1) (2000) 508.

[94] T. A. Gardiner, J. M. Stone, An unsplit Godunov method for ideal MHD via constrained transport, Journal of Computational Physics 205 (2) (2005) $1080 \quad 509-539$.

[95] R. Käppeli, S. Whitehouse, S. Scheidegger, U.-L. Pen, M. Liebendörfer, FISH: A three-dimensional parallel magnetohydrodynamics code for astrophysical applications, The Astrophysical Journal Supplement Series 195 (2) (2011) 20.

1085 [96] J. M. Stone, T. Gardiner, A simple unsplit Godunov method for multidimensional MHD, New Astronomy 14 (2) (2009) 139-148. 